\title{
Existence of nonlinear normal modes of symmetric Hamiltonian systems
}

\author{
James Montaldi, Mark Roberts and Ian Stewart \\ Mathematics Institute, University of Warwick, Coventry CV4 7AL, UK
}

Received 22 November 1988, in final form 15 January 1990

Accepted by R S Mackay

\begin{abstract}
We analyse the existence of nonlinear normal modes of a (nonlinear) Hamiltonian system, i.e. periodic solutions that approximate periodic solutions of the system linearised around an elliptic (and semisimple) equilibrium point. In particular we consider systems with symmetry, including time-reversible symmetry which involves an antisymplectic operator. The general form for such a system contains free parameters (Taylor series coefficients), and our aim is to calculate how the number of nonlinear normal modes varies with these parameters.

We employ a combination of traditional dynamical methods, such as Birkhoff normal form, and more recent results from singularity theory. It is well known that the existence of nonlinear normal modes is equivalent to the existence of a solution to a variational problem on a loop space. We use the splitting lemma (rather than the more usual Liapunov-Schmidt reduction) to convert this variational problem to one in finite dimensions, and relate the result to the Birkhoff normal form. The results are applied to systems whose symmetry group is the dihedral group $\mathbf{D}_{n}$, either with or without time-reversal symmetry.

For the example of $\mathbf{D}_{n}$ symmetry we use ad hoc methods to prove that all nonlinear normal modes have been obtained. We also describe a more systematic method, applying further machinery from singularity theory. We show that under suitable conditions results obtained by Damon on topological determinacy of quasihomogeneous singularities can be used to justify truncating the Birkhoff normal form at an appropriate degree. We describe the use of geometric criteria to verify the requisite non-degeneracy conditions.

In order to show that the calculations involved in this method are tractable, we discuss an example: the $\mathbf{Z}_{2}$-symmetric time-reversible 1:1 resonance. This class of problems includes as a special case the planar spring pendulum at $1: 1$ resonance. By computing the Birkhoff normal form of the resonant spring pendulum to fourth order, we show that this system, when non-degenerate, has either 2,4 or 6 nonlinear normal modes, the number depending on a single quantity which can be expressed in terms of the cubic and quartic spring nonlinearities. This confirms results obtained by Kummer and establishes them rigorously for systems not in exact Birkhoff normal form. We also observe that the calculations for the $\mathbf{Z}_{2}$-symmetric time-reversible 1:-1 resonance are essentially identical, except when establishing which periodic solutions are real.
\end{abstract}

AMS classification scheme numbers: 14B05, 34C15, 58F05, 58F10, 58F14, 58F22, 58F30 


\section{Introduction}

If a Hamiltonian system in equilibrium is slightly displaced, then it may exhibit various kinds of dynamic behaviour, among which periodic oscillations are the simplest and most basic. In a linear Hamiltonian system such oscillations are called normal modes. In nonlinear Hamiltonian systems there can exist families of periodic oscillations that tend towards a normal mode of the linearisation of the system around its equilibrium: these are the nonlinear normal modes. In 1895 Liapunov published his celebrated centre theorem, which in this language asserts the existence of nonlinear normal modes corresponding to each simple non-resonant eigenvalue of the linearisation, see Abraham and Marsden (1978) $\mathrm{p}$ 498. This result was generalised by Weinstein $(1973,1978)$ and Moser (1976) to permit multiple eigenvalues and resonance, under the hypothesis that the quadratic part $\mathrm{H}_{2}$ of the Hamiltonian is definite on each resonance space. The main results of this paper require the linear part of the Hamiltonian vector field to be semisimple, which in particular is the case when $\mathrm{H}_{2}$ is definite on each resonance space.

If the Hamiltonian is invariant under a (compact Lie) group of symmetries then multiple eigenvalues can occur generically. In Montaldi et al (1988) we obtained an analogue of the Weinstein-Moser theorem for symmetric Hamiltonians, proving the existence, under suitable hypotheses, of nonlinear normal modes that break the spatio-temporal symmetry in specified ways. We also considered the linearised stability of such solutions, showing that strong symmetry constraints arise. Indeed, for some classes of solutions, which we called cyclospectral, symmetry can force linearised stability independently of the precise nonlinearity.

That paper left two fundamental questions unanswered.

(a) Existence. Is it possible to find all nonlinear normal modes of a given Hamiltonian system with symmetry?

(b) Stability. Can the linearised stability of these nonlinear normal modes be calculated?

In this paper we develop methods to answer the existence question (a), and show that the calculations involved are tractable for some symmetry groups. In other words, we establish effective existence and non-existence theorems for nonlinear normal modes in symmetric Hamiltonian systems. At the same time we extend the existing methods to permit time-reversal symmetry. Such symmetry is common in applications, but requires technical changes because the time-reversal operator is antisymplectic rather than symplectic. Our approach here is a continuation of the work of Vanderbauwhede (1982).

Throughout we adopt the viewpoint that the Hamiltonian to be studied is the most general function that is invariant under the given group of symmetries. This means that its Taylor expansion contains free parameters, the coefficients of particular terms, and our results are expressed in terms of these coefficients. The following paper, Montaldi et al (1990), develops the same methods further, providing an answer to the stability question (b).

Our approach to nonlinear normal modes is based on a standard method: transformation into Birkhoff normal form. This applies a symplectic change of coordinates to put the original Hamiltonian $H$ into the form

$$
\hat{H}=H_{2}+H_{k}+\tilde{H}
$$


where $\mathrm{H}_{2}$ is the quadratic part of $H, H_{k}$ is of degree $\leqslant k$ and has extra symmetry determined by the linear flow of $H_{2}$, and $\tilde{H}$ is of order $>k$ and has only the original symmetry. If (1.1) is truncated at degree $k$ to yield a Hamiltonian $\hat{H}_{k}=H_{2}+H_{k}$, then the nonlinear normal modes of $\hat{H}_{k}$ correspond bijectively to solutions of the variational problem

$$
D_{x}\left(-\tau H_{2}+H_{k}\right)=0
$$

where $\tau$ is a parameter representing the period.

The main new ingredient in this paper is theorem 5.1. This gives precise conditions under which the solutions of (1.2) are rigorously proved to correspond, not only to nonlinear normal modes of the truncated Hamiltonian $\hat{H}_{k}$, but to nonlinear normal modes of the original Hamiltonian $H=H_{2}+H_{k}+\tilde{H}$. To do this we use results from singularity theory, namely a version of the splitting lemma due to Magnus (1980), and a theorem of Damon (1988) on finite topological determinacy. Theorem 5.1 applies only to Hamiltonians for which $H_{k}$ is homogeneous, but we anticipate that with a little further effort the results of Damon (1988) can be applied to more general cases.

In specific cases, it can sometimes be shown by ad hoc means that solutions of (1.2) correspond to nonlinear normal modes of the original Hamiltonian $H$. We consider two examples, each of interest in its own right, to show how these ad hoc methods and theorem 5.1 apply in practice.

Our aims in this paper are similar to those of Duistermaat (1984) and van der Meer (1985). Those authors also use singularity theory to give rigorous proofs of the existence of nonlinear normal modes. However, the details of the approaches are different. Firstly, as remarked below, we use the splitting lemma rather than Liapunov-Schmidt reduction. Secondly, we use a completely different method to prove the finite determinacy of truncated normal forms. Roughly speaking, Duistermaat and van der Meer use $C^{l} \mathbf{S}^{1}$-equivariant finite $\mathscr{A}$-determinacy of the mapping $\left(H_{k}, H_{2}\right)$ into $\mathbb{R}^{2}$, while we use $C^{0} \mathbf{S}^{1}$-equivariant finite $\mathscr{H}$-determinacy of an auxiliary mapping $D_{x}\left((-\tau+J \lambda) H_{2}+H_{\kappa}\right)$ where $J$ is the symplectic involution and $\lambda$ a new parameter. Note that the singularities of $\left(H_{k}, H_{2}\right)$ correspond to solutions of $D_{x}\left((-\tau+J \lambda) H_{2}+H_{k}\right)=0$ when $\lambda=0$. In general, finite $\mathscr{K}$-determinacy is easier to calculate than finite $\mathscr{A}$-determinacy, while the use of topological finite determinacy helps overcome problems associated with moduli so lowering the codimension. However, our use of these techniques is perhaps at the cost of theoretical elegance.

In more detail, the contents of the paper are as follows.

In section 2 we collect and summarise the necessary results from the theory of Birkhoff normal forms. These are scattered throughout the literature, but not always in the precise form that we need. We have therefore given a systematic account, emphasising symmetry aspects, but have suppressed proofs. We include an account of how the results must be modified to incorporate time-reversal symmetry which, as remarked above, does not preserve the symplectic structure.

The standard method for reducing the search for nonlinear normal modes to a finite-dimensional problem is Liapunov-Schmidt reduction, see Moser (1976) and Duistermaat (1984). However, this does not obviously preserve the variational structure inherent in the problem, and instead in section 3 we use a version of the splitting lemma due to Magnus (1980). We discuss connections with Birkhoff normal form. Again we emphasise symmetry and include the time-reversible case. A suitable reduction method, other than Birkhoff normal form, is important here 
because it provides a rigorous approach to nonlinear normal modes, not directly involving truncation of the Hamiltonian. We make use of symmetry to distinguish between various types of periodic trajectory, such as standing and rotating waves. The presence of time-reversal symmetry allows us to characterise brake orbits (see Churchill et al 1983) group theoretically.

Section 4 is devoted to an analysis of Hamiltonian systems with dihedral group symmetry $\mathbf{D}_{n}$. In Montaldi et al (1988) we proved the existence of three types of symmetry-breaking nonlinear normal modes in systems whose Hamiltonian is invariant under the standard action of $\mathbf{D}_{n}$. Here we show, by an ad hoc argument, that there are no others. We also consider time-reversible Hamiltonian systems with $\mathbf{D}_{n}$ symmetry.

The central theoretical result is treated in section 5 , which addresses the question of existence and non-existence of nonlinear normal modes, using singularitytheoretic methods. The main result, theorem 5.1, provides a rigorous justification (under appropriate conditions) for the procedure of truncating the Birkhoff normal form in (1.2), by applying a result of Damon (1988). Essentially, Damon's result states that if a weighted homogeneous singularity is finitely determined at some order under smooth equivalence, then it is topologically finitely determined at the lowest significant order, under topological equivalence. Finite determinacy means that the zero set does not change its qualitative form after truncation at the appropriate order. The success of the method relies on applying Damon's result, not to the mapping $D_{x}\left(-\tau H_{2}+H_{k}\right)$ in (1.2), but to the related auxiliary mapping $D_{x}\left((-\tau+J \lambda) H_{2}+H_{k}\right)$. This change is needed to achieve finite determinacy.

The use of theorem 5.1 is illustrated in section 6 , which analyses the general time-reversible 1:1 resonance with $\mathbf{Z}_{2}$ symmetry. For tactical reasons we use geometric criteria (Roberts 1986) rather than the more usual algebraic 'tangent space' criteria, to check the hypotheses of theorem 5.1. We show that generically there exist two, three or four different symmetry types of nonlinear normal mode. Theorem 6.3 states precise existence (and stability) criteria for these in terms of the Birkhoff normal form truncated at fourth order. This example shows that the hypotheses of theorem 5.1 are verifiable in practice.

A specific system having $\mathbf{Z}_{2}$ and time-reversal symmetry is the planar spring pendulum. For suitable parameter values (such as the spring constant) this can be made to vibrate in $1: 1$ resonance between its spring and pendulum modes. We choose this resonance because of its theoretical interest: other resonances can also occur and can be analysed in a similar way. By computing the reduction to Birkhoff normal form we study the nonlinear normal modes of the spring pendulum at $1: 1$ resonance, providing rigorous confirmation of the results of Kummer (1986) and extending them slightly.

\section{Birkhoff normal form}

We begin by giving a brief summary of the results we shall need from the theory of Birkhoff normal forms of symmetric Hamiltonian systems. Our account is adapted from Churchill et al (1983), van der Meer (1985), Elphick et al (1987) and Vanderbauwhede (1989), to which we refer for further discussion and full proofs. The emphasis is on systems with symmetry, including time-reversal symmetry.

The results of this paper are local. We can therefore choose a convenient local coordinate system on the underlying symplectic manifold $P$, in which the equi- 
librium point $p$ is the origin. This lets us identify $P$ with $T_{0} P$. Without loss of generality we may take $P=\mathbb{C}^{n}$ and define the Hamiltonian structure as follows. Let $\{z, w\}=\sum_{j=1}^{n} z_{j} \bar{w}_{j}$ denote the standard Hermitian form on $\mathbb{C}^{n}$. Then $(z, w)=$ $\operatorname{Re}\{z, w\}$ and $[z, w]=\operatorname{Im}\{z, w\}$ are an inner product and symplectic form, respectively. Let $\Gamma$ be a compact Lie group acting unitarily, and hence orthogonally and symplectically, on $\mathbb{C}^{n}$. The space $\mathbb{C}^{n}$ together with this unitary action will be denoted by $V$. Let $H: V \times \Lambda \rightarrow \mathbb{R}$ be a smooth real-valued $\Gamma$-invariant function on $V$, depending smoothly on parameters $\lambda$ in the Banach space $\Lambda$. The associated Hamiltonian differential equation is given by

$$
\dot{z}_{j}=\mathrm{i} \frac{\partial H}{\partial \bar{z}_{j}} \quad j=1, \ldots, n
$$

or, more briefly, $\dot{z}=\mathrm{i} D_{\bar{z}} H(z, \lambda)$. If

$$
z_{j}=\frac{1}{\sqrt{2}}\left(p_{j}+\mathrm{i} q_{j}\right)
$$

then

$$
\frac{\partial}{\partial \bar{z}_{j}}=\frac{1}{\sqrt{2}}\left(\frac{\partial}{\partial p_{j}}+\mathrm{i} \frac{\partial}{\partial q_{j}}\right)
$$

and (2.1) corresponds to the usual form of Hamilton's equations:

$$
\dot{p}_{j}=-\frac{\partial H}{\partial q_{j}} \quad \dot{q}_{j}=\frac{\partial H}{\partial p_{j}} \quad j=1, \ldots, n .
$$

A Hamiltonian system is time reversible if $H(-p, q)=H(p, q)$ or equivalently, in the above complex coordinates, if $H(-\bar{z})=H(z)$. While other definitions of time-reversibility are conceivable, there is little loss of generality in assuming this form. We denote the time-reversal involution $z \rightarrow-\bar{z}$ by $\rho$, and the group generated by this operation by $\mathbf{Z}_{2}^{\rho}$. The action of $\rho$ is antisymplectic, i.e. $[\rho x, \rho y]=-[x, y]$. The group generated by $\Gamma$ and $\mathbf{Z}_{2}^{\rho}$ is the semidirect product $\Gamma \rtimes \mathbf{Z}_{2}^{\rho}$. If $\Gamma$ acts on configuration space and dually on momentum space then $\Gamma$ and $\mathbf{Z}_{2}^{p}$ commute, and we have the direct product $\Gamma \times \mathbf{Z}_{2}^{\rho}$. Define

$$
\hat{\Gamma}= \begin{cases}\Gamma \rtimes \mathbf{Z}_{2}^{\rho} & \text { if } H \text { is time reversible } \\ \Gamma & \text { otherwise. }\end{cases}
$$

suppose $H(0, \lambda)=0=D_{\bar{z}} H(0, \lambda)$, so that (2.1) has an equilibrium point at $0 \in V$ for all $\lambda$. Let $H_{2}=j^{2} H(0)$. Here, and subsequently, we use $j^{k} H(\lambda)$ to denote the $k$-jet (i.e. the Taylor series expansion to degree $k$ ) at 0 of $H(, \lambda)$, considered as a function of $z$ alone. Thus $H_{2}$ is a $\hat{\Gamma}$-invariant quadratic polynomial in $z$, which is independent of $\lambda$.

Linearising (2.1) at $0 \in V$ gives

$$
\dot{z}=L \cdot z
$$

where $L=\mathrm{i} D_{z} D_{\bar{z}} H(0,0)$ is an infinitesimally symplectic linear map; i.e. it satisfies

$$
[L \cdot v, w]+[v, L \cdot w]=0 \quad \text { for all } v, w \in V .
$$

The transpose of $L$, denoted $L^{\mathrm{T}}$, generates a one-parameter group $\left\{\exp \left(t L^{\mathrm{T}}\right): t \in \mathbb{R}\right\}$ of symplectic transformations of $V$. The closure of this group; denoted $\mathbf{S}$, is an Abelian Lie group of symplectic transformations of $V$, see Elphick et al (1987) and Vanderbauwhede (1989). 
Since $\Gamma$ acts symplectically and $H$ is $\Gamma$ invariant, it follows that both $L$ and $\mathrm{S}$ commute with the $\Gamma$-action on $V$. However, if $H$ is time reversible, then $L$ anticommutes with $\rho$, i.e. $L \circ \rho=-\rho \circ L$. (Alternatively, we may say that $L$ commutes with $\rho$ if we define the action of $\rho$ on the target of $L$ to be $-I$ times that on the source). Therefore, if $s \in \mathbf{S}$ then $\rho^{-1} s \rho=s^{-1}$. If $H$ is time reversible, let $\hat{\mathbf{S}}$ be the semidirect product of $\mathbf{S}$ and $\mathbf{Z}_{2}^{\rho}$ defined by this action of $\mathbf{Z}_{2}^{\rho}$ on $\mathbf{S}$. Otherwise let $\hat{\mathbf{S}}=\mathbf{S}$.

If, as we shall assume, $L$ is semisimple (diagonalisable over $\mathbb{C}$ ) and has imaginary eigenvalues, then $\mathbf{S}$ is isomorphic to the $(n-k)$-dimensional torus $\mathbf{T}^{n-k}$, where $k$ is the number of independent resonance conditions between the eigenvalues of $L$. In general, however; $\mathbf{S}$ need not be compact. If $L$ has imaginary eigenvalues, is semisimple, and is 'fully resonant', i.e. all the eigenvalues are integer multiples of one imaginary number, then $\mathbf{S} \cong \mathbf{S O}(2)$; and for time-reversible systems, $\hat{\mathbf{S}} \cong \mathbf{O}(2)$.

We now state the Birkhoff normal form theorem for symmetric Hamiltonian systems.

Theorem 2.1. For any integer $k \geqslant 2$ there exists a neighbourhood $\Lambda_{k}$ of 0 in $\Lambda$, a $\hat{\Gamma}$-invariant neighbourhood $U_{k}$ of 0 in $V$ and a $\hat{\Gamma}$-equivariant mapping $\Psi: U_{k} \times \Lambda_{k} \rightarrow$ $V$ with $D_{\bar{z}} \Psi(0,0)=I$ and such that, for each $\lambda \in \Lambda_{k}$ :

(i) $\Psi(, \lambda)$ is a $\hat{\Gamma}$-equivariant symplectic diffeomorphism of $U_{k}$ onto its image, fixing 0 ;

(ii) $j^{k}\left(H \circ \Psi-H_{2}\right)(\lambda)$ is invariant under the $\Gamma \rtimes \hat{\mathbf{S}}$-action on $V$.

In particular the Birkhoff normal form of a time-reversible system is time-reversible.

\section{Outline of the proof.}

We concentrate on the main points of the time-reversible parameter-independent case. Details can be found in the references given above. Let $\mathscr{E}$ denote the space of germs at 0 of $\hat{\Gamma}$-invariant smooth real-valued functions $f$ on $V$ and $P_{k}$ the subspace of germs of $\hat{\Gamma}$-invariant real-valued homogeneous polynomials of degree $k$ in $z_{j}$ and $\bar{z}_{j}$. The space $\mathscr{E}$ decomposes as $\mathscr{E}^{+} \oplus \mathscr{E}^{-}$where $\mathscr{E}^{+}$consists of functions invariant under $\mathbf{Z}_{2}^{\rho}$ and $\mathscr{E}^{-}$of anti-invariant functions:

$$
\mathscr{E}^{+}=\{f: f(\rho \cdot z)=f(z)\}, \quad \mathscr{E}^{-}=\{f: f(\rho \cdot z)=-f(z)\} .
$$

There is a corresponding decomposition of $P_{k}$.

The Poisson bracket of two germs, $f$ and $h$, in $\mathscr{E}$ is given by

$$
\{h, f\}=\operatorname{Im}\left(\sum_{j=1}^{n} \frac{\partial h}{\partial \bar{z}_{j}} \frac{\partial f}{\partial z_{j}}\right) .
$$

For $h \in \mathscr{E}$ define ad $h: \mathscr{E} \rightarrow \mathscr{E}$ by ad $h(f)=\{h, f\}$. If $h \in \mathscr{E}^{-}$then the operator ad $h$ preserves the subspaces $\mathscr{E}^{ \pm}$, while if $h \in \mathscr{C}^{+}$then it interchanges them. Let $\operatorname{ad}_{+} h=\left.\operatorname{ad} h\right|_{\mathscr{C}^{+}}$and $\operatorname{ad}_{-} h=\left.\operatorname{ad} h\right|_{\mathscr{C}^{-}}$. If $h \in P_{l}$ then $\operatorname{ad} h: P_{k} \rightarrow P_{k+l-2}$ and, in particular, ad $H_{2}: P_{k} \rightarrow P_{k}$. We also define an operator exp ad $h: \mathscr{E} \rightarrow \mathscr{E}$ by

$$
\exp \operatorname{ad} h=\sum_{r=0}^{\infty} \frac{1}{r !} \operatorname{ad}^{r}(h) .
$$

Thus ad $h$ is the Hamiltonian vector field associated with $h$. Then $\exp (t \operatorname{ad} h)$ is the time- $t$ flow whose action on the underlying space $V$ is given in coordinates by lemma 2.2 below. If $H \in \mathscr{E}^{+}$then the corresponding vector field and time-t flow are time-reversible, while if $h \in \mathscr{E}^{-}$they are $\mathbf{Z}_{2}^{\rho}$-equivariant. 
Lemma 2.2. If $f \in \mathscr{E}^{+}, h \in \mathscr{E}^{-}$and $h$ has a critical point at $0 \in V$ then $\exp$ ad $h(f)=$ $f \circ \Psi$ where $\psi$ is the $\hat{\Gamma}$-equivariant symplectic diffeomorphism germ at $0 \in V$ defined by

$$
\psi_{j}(z)=\exp \operatorname{ad} h\left(z_{j}\right) .
$$

Define the fixed-point space $\operatorname{Fix}\left(\mathbf{S} ; P_{k}\right)=\left\{p \in P_{k}: \sigma p=p \forall \sigma \in \mathbf{S}\right\}$. The group $\mathbf{S}$ acts on each $P_{k}$ in such a way that the spaces Fix $\left(\mathbf{S} ; P_{k}\right)$ are the spaces of germs at 0 of $\Gamma \rtimes \hat{\mathbf{S}}$-invariant homogeneous polynomials of degree $k$ in $z_{j}$ and $\bar{z}_{j}, j=1, \ldots, n$.

The following lemma is the equivariant time-reversibe Hamiltonian analogue of the central idea of Elphick et al (1987).

Lemma 2.3.

$$
P_{k}=\operatorname{Im}\left(\operatorname{ad} H_{2}\right) \oplus \operatorname{Fix}\left(\mathbf{S} ; P_{k}\right) .
$$

Proof. Let $H_{2}^{\mathrm{T}}$ denote the quadratic polynomial Hamiltonian which generates the flow $\exp \left(t L^{\mathrm{T}}\right)$ on $\mathbb{C}^{n}$. Then

$$
\operatorname{Fix}\left(\mathbf{S} ; P_{k}\right)=\left\{f \in P_{k}:\left\{H_{2}^{\mathrm{T}}, f\right\}=0\right\}=\operatorname{ker} \operatorname{ad} H_{2}^{\mathrm{T}} \cap P_{k} .
$$

Let $Q_{k-1}$ denote the space of homogeneous vector fields of degree $k-1$ on $\mathbb{C}^{n}$ and $X_{f}$ the vector field generated by $f \in P_{k}$. If $h \in P_{k}, f \in P_{l}$ then $X_{\{h, f\}}=\llbracket X_{h}, X_{f} \rrbracket$ where $\llbracket, \rrbracket$ is the usual Lie bracket of vector fields. In particular $X_{\operatorname{ad} H_{2}(f)}=\operatorname{ad} L \cdot\left(X_{f}\right)$ where $\operatorname{ad} L$ is the usual adjoint action of $L$ on vector fields.

Elphick et al (1987) define an inner product $\langle$,$\rangle on Q_{k-1}$ with the property that $(\operatorname{ad} L)^{\mathrm{T}}=\operatorname{ad}\left(L^{\mathrm{T}}\right)$ with respect to this inner product. See also Golubitsky et al (1988) p. 288. Define an inner product $\langle$, $\rangle$ on $P_{k}$ by

Then

$$
\langle h, f\rangle=\left\langle X_{h}, X_{f}\right\rangle \text {. }
$$

$$
\begin{gathered}
\left\langle\operatorname{ad} H_{2}(h), f\right\rangle=\left\langle X_{\operatorname{ad} H_{2}(h)}, X_{f}\right\rangle=\left\langle\operatorname{ad} L\left(X_{h}\right), X_{f}\right\rangle=\left\langle X_{h}, \operatorname{ad} L^{\mathrm{T}}\left(X_{f}\right)\right\rangle \\
=\left\langle X_{h}, X_{\operatorname{ad} H_{2}^{\mathrm{T}}(f)}\right\rangle=\left\langle\left\langle h, \operatorname{ad} H_{2}^{\mathrm{T}}(f)\right\rangle .\right.
\end{gathered}
$$

Thus $\left(\operatorname{ad~} H_{2}\right)^{\mathrm{T}}=\operatorname{ad}\left(H_{2}^{\mathrm{T}}\right)$. Now $P_{k}=\operatorname{Im}\left(\operatorname{ad} H_{2}\right) \oplus \operatorname{ker}\left(\operatorname{ad} H_{2}\right)^{\mathrm{T}}$ and $\operatorname{ker}\left(\operatorname{ad} H_{2}\right)^{\mathrm{T}}=$ $\left.\operatorname{ker} \operatorname{ad}\left(H_{2}^{\mathrm{T}}\right)=\operatorname{Fix}\left(\mathbf{S} ; P_{k}\right)\right)$. The result follows by taking intersections with $P_{k}^{+}$.

The proof of theorem 2.1 is by induction on $k$. In the absence of parameters it is trivially true for $k=2$. Now write $j^{k} H=H_{2}+\ldots+H_{k-1}+\hat{H}_{k}+\tilde{H}_{k}$ where

$$
\begin{aligned}
& H_{j} \in \operatorname{Fix}\left(\mathbf{S} ; P_{j}\right) \quad j=3, \ldots, k-1 \\
& \hat{H}_{k} \in \operatorname{Fix}\left(\mathbf{S} ; P_{k}\right) \quad \tilde{H}_{k} \in \operatorname{Im}\left(\operatorname{ad} H_{2}\right) \subset P_{k} .
\end{aligned}
$$

Choose $L_{k} \in P_{k}$ such that ad $H_{2}\left(L_{k}\right)=\tilde{H}_{k}$. Then

$j^{k}\left[\exp\right.$ ad $\left.L_{k}(H)\right]=H_{2}+\ldots+H_{k-1}+\hat{H}_{k}+\tilde{H}_{k}+\left\{L_{k}, H_{2}\right\}$

$$
=H_{2}+\ldots+H_{k-1}+\hat{H}_{k}
$$

since $\left\{L_{k}, H_{2}\right\}=-\left\{H_{2}, L_{k}\right\}=-\tilde{H}_{k}$. The inductive step is completed by applying lemma 2.2 .

The parameter-dependent case can be proved as in Vanderbauwhede (1989). The essential point is that if $H$ can be put into Birkhoff normal form to degree $k$ when $\lambda=0$ then the implicit function theorem can be used to solve the necessary equations to put it into Birkhoff normal form to degree $k$ for all $\lambda$ in a neighbourhood $\Lambda_{k}$ of 0 . 
We now give a more explicit description of the action of $\mathbf{S}$ on $V$ when $L$ is semisimple with non-zero imaginary eigenvalues. In this case $L^{\mathrm{T}}=-L$. We describe the action in terms of the resonance subspaces $V_{\alpha_{j}}$, defined as follows. For any real non-zero $\alpha$ let $V_{\alpha} \subset V$ be the fixed-point space of $\exp [(2 \pi / \alpha) L]$, i.e. the set of all points that lie on $(2 \pi / \alpha)$-periodic trajectories of the linearised equation (2.2). If $n \in \mathbb{N}$ then $V_{n \alpha} \subset V_{\alpha}$. Define two eigenvalues of $L$ to be equivalent if their ratio is in $\mathbb{Q}$. Suppose that there are $r$ equivalence classes. Let $\alpha_{j}$ be such that every element of the $j$ th equivalence class is an integer multiple of $i \alpha_{j}$ and $\alpha_{j}$ is maximal subject to that property. Then $V_{\alpha_{j}}$ is a resonance subspace and

$$
V=\bigoplus_{j=1}^{r} V_{\alpha_{j}}
$$

This decomposition of $V$ can also be characterised by the following two conditions:

(i) each $V_{\alpha_{j}}$ is an $L$-invariant subspace of $V$ and the eigenvalues of the restriction $\left.L\right|_{v_{\alpha_{j}}}$ are (non-zero) integer multiples of $i \alpha_{j}$;

(ii) the $\alpha_{j}$ are pairwise linearly independent over the field of rational numbers. The resonance subspaces $V_{\alpha_{j}}$ are $\Gamma \rtimes \hat{\mathbf{S}}$-invariant symplectic subspaces of $V$. Let $\pi_{j}$ denote the induced projection from $V$ onto $V_{\alpha_{j}}$ and define a $\Gamma$-equivariant linear symplectic map $L_{j}: V \rightarrow V$ by $L_{j}=L \circ \pi_{j}$. Let

$$
\mathbf{S}_{j}=\overline{\left\{\exp t L_{j}^{\mathrm{T}}: t \in \mathbb{R}\right\}} \text {. }
$$

Then $\mathbf{S}_{j}$ is isomorphic to the circle group $\mathbf{S}^{1}$ and $\mathbf{S} \cong \mathbf{S}_{1} \times \ldots \times \mathbf{S}_{r} \cong \mathbf{T}$. Moreover, $\mathbf{S}_{j}$ acts trivially on $V_{\alpha_{k}}$ for $k \neq j$, but non-trivially on $V_{\alpha_{j}}$. If $\operatorname{dim} V_{\alpha_{j}}=n(j)$ and the eigenvalues of $L_{j}$ are $\pm \mathrm{i} p_{1} \alpha_{j}, \ldots, \pm \mathrm{i} p_{n(j)} \alpha_{j}$ where $p_{i} \in \mathbb{Z} \backslash\{0\}$, then $V_{\alpha_{j}}$ can be identified with $\mathbb{C}^{n(j)}$ in such a way that the action of $\mathbf{S}_{j}$ becomes

$$
\theta \cdot\left(z_{1}, \ldots, z_{n_{j}}\right)=\left(\exp \left(\mathrm{i} p_{1} \theta\right) z_{1}, \ldots, \exp \left(\mathrm{i} p_{n(j)} \theta\right) z_{n(j)}\right)
$$

As a consequence of this decomposition we obtain the following result.

Corollary 2.4. Let $H: V \rightarrow \mathbb{R}$ be a $\Gamma$-invariant Hamiltonian on $V$ with $D_{\bar{z}} H(0)=0$ and suppose that $L=\mathrm{i} D_{z} D_{\bar{z}} H(0)$ is semisimple with non-zero purely imaginary eigenvalues. If $V_{\alpha_{j}}$ is a resonance subspace for $L$ and $H$ is in Birkhoff normal form to degree $k$, then the gradient and Hamiltonian vector fields generated by $j^{k} H$ are tangent to $V_{\alpha_{j}}$ when restricted to $V_{\alpha_{j}}$ and are equal to the corresponding vector fields generated by $j^{k}\left(\left.H\right|_{\alpha_{\alpha_{j}}}\right)$. The flows of these vector fields leave $V_{\alpha_{j}}$ invariant.

Proof. If $K$ is a subgroup of the group $G$ then any $G$-equivariant dynamical system leaves $\operatorname{Fix}(K)$ invariant. The corollary is obtained by applying this result to the S-invariant dynamical system given by $j^{k} H$ with $K=\prod_{k \neq j} \mathbf{S}_{k}$, noting that $\operatorname{Fix}(K)=V_{\alpha_{j}}$.

We end this section by giving two operators which are useful in practical computations of Birkhoff normal forms, see, for example, Vanderbauwhede (1989). We continue to suppose that $L$ is semisimple and has imaginary eigenvalues, so that $\mathbf{S}$ is a torus. The first operator is the projection $\pi: P_{k} \rightarrow \operatorname{Fix}\left(\mathbf{S} ; P_{k}\right)$ with kernel $\operatorname{Im}\left(\operatorname{ad} H_{2}\right)$; this is given explicitly by

$$
\pi(f)=\lim _{\sigma \rightarrow \infty} \frac{1}{\sigma} \int_{0}^{\sigma} f\left(\exp \left(t L^{\mathrm{T}}\right) \cdot z\right) \mathrm{d} t
$$


and is used to split $H_{k}$ into $\hat{H}_{k}+\tilde{H}_{k}$. The second operator is $\eta: \operatorname{Im}\left(\operatorname{ad} H_{2}\right) \cap P_{k} \rightarrow P_{k}$ defined by

$$
\eta(f)=\lim _{\sigma \rightarrow \infty} \frac{1}{\sigma} \int_{0}^{\sigma} \int_{0}^{t} f\left(\exp \left(s L^{\mathrm{T}}\right) \cdot z\right) \mathrm{d} s \mathrm{~d} t .
$$

This satisfies ad $H_{2}^{\circ} \eta=I-\pi$ and so, if $f \in \operatorname{Im}$ ad $H_{2}$ then

$$
\text { ad } H_{2}(\eta(f))=(I-\pi)(f)=f \text {. }
$$

Thus $\eta$ is used to calculate $L_{k}$ such that ad $H_{2}\left(L_{k}\right)=\tilde{H}_{k}$. If $L$ is fully resonant, i.e. $V$ itself is the only resonance subspace, then $\mathbf{S} \cong \mathbf{S}^{1}$ and these formulae for $\pi$ and $\eta$ can be replaced by

$$
\pi(f)=\frac{1}{2 \pi} \int_{0}^{2 \pi} f(\theta \cdot z) \mathrm{d} \theta \quad \eta(f)=\frac{1}{2 \pi} \int_{0}^{2 \pi} \int_{0}^{\varphi} f(\theta \cdot z) \mathrm{d} \theta \mathrm{d} \varphi .
$$

\section{Reduction via the splitting lemma}

The usual procedure for finding periodic trajectories near an equilibrium point (in, for example, a Hopf bifurcation) is to set up an equation on a loop space and then reduce it to a finite-dimensional equation using Liapunov-Schmidt reduction. For Hamiltonian systems the equation on a loop space has a variational structure which is not obviously preserved by Liapunov-Schmidt reduction. We therefore prefer to use a version of the splitting lemma due to Magnus (1980), which does preserve the variational structure.

If $V$ is a finite-dimensional real vector space, let $\mathscr{C}^{r}\left(\mathbf{S}^{1}, V\right)$ denote the Banach space of $C^{r}$ maps $\mathbf{S}^{1} \rightarrow V$ with the $C^{r}$ norm. Let $H$ be a Hamiltonian function on a phase space $P$ with symplectic form $\omega$. Assume that the linearised vector field defined by $\mathrm{H}$ is semisimple; in particular this is the case if the quadratic part $\mathrm{H}_{2}$ of $\mathrm{H}$ is definite on each resonance space. As usual, identify $P$ with $T_{0} P$ and denote both by $V$. Define the action functional $S: \mathscr{C}^{1}\left(\mathbf{S}^{1}, V\right) \times \mathbb{R} \rightarrow \mathbb{R}$ by

$$
S_{\alpha}(u)=S(u, \alpha)=\alpha \int_{\mathbf{S}^{1}} u^{*} \beta-\int_{0}^{2 \pi} H \circ u(\theta) \mathrm{d} \theta
$$

where $\beta$ is a 1 -form satisfying $\mathrm{d} \beta=\omega$ (for example, we may take $\beta=\sum p_{i} \mathrm{~d} q_{i}$ in the usual Darboux coordinates) and $\mathbf{S}^{1}=\mathbb{R} / 2 \pi \mathbb{Z}$.

Let $X=\mathscr{C}^{1}\left(\mathbf{S}^{1}, V\right)$ and $Y=\mathscr{C}^{0}\left(\mathbf{S}^{1}, V^{*}\right)$, and define $\sigma: Y \rightarrow X^{*}$ to be the continuous linear injection given by

$$
\sigma\left(w^{*}\right)(v)=\int_{0}^{2 \pi} w^{*}(v) \mathrm{d} t \quad \text { for } w^{*} \in Y, v \in X
$$

Define $R_{\alpha}: X \rightarrow Y$ by

$$
R_{\alpha}(u)=\alpha \omega \neg \dot{u}-D H(u)
$$

where $\omega \neg \dot{u}$ is the contraction of $\omega$ with $\dot{u}$ i.e.

$$
(\omega \neg \dot{u})(v)=\omega(\dot{u}, v) \quad \forall v \in V .
$$

Note that $R_{\alpha}(u)=0$ if and only if $z(t) \equiv u(\alpha t)$ is a periodic solution, of period $2 \pi / \alpha$, 
of the Hamiltonian system defined by $H$. We see below that $\sigma R_{\alpha}$ is the differential of $S_{\alpha}$.

Now define $T_{\alpha}: X \rightarrow Y$ by $T_{\alpha}=D R_{\alpha}(0)$, so that

$$
\left.T_{\alpha}(v)=\alpha \omega\right\urcorner \dot{v}-D^{2} H(0)(v,) .
$$

Then $\operatorname{ker} T_{\alpha}$ is precisely the set of $(2 \pi / \alpha)$-periodic solutions of the linearised Hamiltonian equation at $0 \in V$, and so can (and will) be identified with the resonance subspace $V_{\alpha}$ of $V$ defined in section 2 , by identifying each loop $u$ with its initial point $u(0)$.

Proposition 3.1. (i) A loop $u \in \mathscr{C}^{1}\left(\mathbf{S}^{1}, V\right)$ is a critical point of $S_{\alpha}$ if and only if $z(t) \equiv u(\alpha t)$ is a periodic solution, of period $2 \pi / \alpha$, of the Hamiltonian system defined by $H$.

(ii) The null space $\mathcal{N}\left(D^{2} S_{\alpha}(0)\right)$ is equal to $\operatorname{ker} T_{\alpha}$ and can thus be identified with $V_{\alpha}$.

Proof. (i) Let $s \mapsto u_{s}$ be a path in $\mathscr{C}^{1}\left(\mathbf{S}^{1}, P\right)$ with $u_{0}=u$ and $\mathrm{d} u_{s} /\left.\mathrm{d} s\right|_{s=0}=v$. Then

$S_{\alpha}\left(u_{s}\right)=\alpha \int_{\mathbf{S}^{1}} u_{s}^{*} \beta-\int_{0}^{2 \pi} H\left(u_{s}(\theta)\right) \mathrm{d} \theta=\alpha \int_{C_{s}} \boldsymbol{u}_{s}^{*} \omega+\alpha \int_{\mathbf{S}^{1}} u_{0}^{*} \beta-\int_{0}^{2 \pi} H\left(u_{s}(\theta)\right) \mathrm{d} \theta$

where $C_{s}=[0, s] \times \mathbf{S}^{1}$ and $\boldsymbol{u}: C_{s} \rightarrow P$ is given by $\boldsymbol{u}(t, \theta)=u_{t}(\theta)$. The second equality follows by Stokes' theorem.

Thus

$$
S_{\alpha}\left(u_{s}\right)=\alpha \int_{0}^{s} \int_{0}^{2 \pi} \omega\left(\frac{\partial \boldsymbol{u}}{\partial \theta}, \frac{\partial \boldsymbol{u}}{\partial t}\right) \mathrm{d} \theta \mathrm{d} t+\alpha \int_{\mathbf{S}^{1}} u_{0}^{*} \beta-\int_{0}^{2 \pi} H\left(u_{s}(\theta)\right) \mathrm{d} \theta
$$

Differentiating with respect to $s$ at $s=0$ gives

So

$$
D S_{\alpha}(u)(v)=\int_{0}^{2 \pi}(\alpha \omega(\dot{u}(\theta), v(\theta))-D H(u(\theta))) \mathrm{d} \theta .
$$

$$
D S_{\alpha}(u)=\sigma R_{\alpha}(u)
$$

and the critical points of $S_{\alpha}$ are precisely the solutions of $R_{\alpha}(u)=0$. The result follows.

(ii) Differentiating (3.2) at $u=0$ gives $D^{2} S_{\alpha}(0): X \rightarrow X^{*}$ as

$$
\begin{aligned}
D^{2} S_{\alpha} & =\sigma \circ D R_{\alpha}(0) \\
& =\sigma \circ T_{\alpha} .
\end{aligned}
$$

Its null space is therefore $\operatorname{ker} T_{\alpha}$ as required.

Now define

$$
Z_{\alpha}=\left\{z \in X: \int \omega(z, v)=0 \forall v \in V_{\alpha}\right\} \quad W_{\alpha}=\left\{\omega \neg v: v \in V_{\alpha}\right\} \subset Y .
$$

Note that $\sigma W_{\alpha}$ annihilates $Z_{\alpha}$, and since $V_{\alpha}$ is symplectic and finite dimensional, $W_{\alpha}$ can be identified with $V_{\alpha}^{*}$. It follows that $X^{*}=\sigma\left(W_{\alpha}\right) \oplus V_{\alpha}^{\perp}$. 
Lemma 3.2. (i) $Y=\operatorname{Im}\left(T_{\alpha}\right) \oplus W_{\alpha}$; (ii) $X=V_{\alpha} \oplus Z_{\alpha}$.

Proof. Since $\sigma$ is injective and continuous, the decomposition of $X^{*}$ implies that $Y=W_{\alpha} \oplus \sigma^{-1}\left(V_{\alpha}^{\perp}\right)$. To establish (i) it is sufficient to show that $\operatorname{Im}\left(T_{\alpha}\right)=\sigma^{-1}\left(V_{\alpha}^{\perp}\right)$, where $V_{\alpha}^{\perp} \equiv\left(\operatorname{ker} T_{\alpha}\right)^{\perp}=\operatorname{ann}\left(V_{\alpha}\right) \subset X^{*}$.

First we show that $Y \subset \operatorname{Im}\left(T_{\alpha}\right) \oplus W_{\alpha}$. If $v \in V_{\alpha}$ then $\int\left\langle T_{\alpha} u, v\right\rangle=\int\left\langle u, T_{\alpha} v\right\rangle=0$ for all $u$, because $T_{\alpha}=D^{2} S_{\alpha}(\alpha)$ is symmetric.

Next we show that $Y \supset \operatorname{Im}\left(T_{\alpha}\right) \oplus W_{\alpha}$. Let $y \in Y$ be such that $\sigma(y) \in V_{\alpha}^{\perp}$, i.e. $\int\langle y, v\rangle=0$ for all $v \in \operatorname{ker} T_{\alpha}$. We want to solve $T_{\alpha} u=y$. Decompose $y=y_{1}+y_{2}$, with $y_{1}(t) \in\left(V_{\alpha}^{\prime}\right)^{\perp} \subset V^{*}$ and $y_{2}(t) \in V_{\alpha}^{\perp} \subset V^{*}$, where $V_{\alpha}^{\prime}$ is the unique $L$-invariant complement to $V_{\alpha}$ in $V$. We first solve $T_{\alpha} u_{1}=y_{1}$ for $u_{1} \in \mathscr{C}^{1}\left(\mathbf{S}^{1}, V_{\alpha}\right)$, and then solve $T_{\alpha} u_{2}=y_{2}$ for $u_{2} \in \mathscr{C}^{1}\left(\mathbf{S}^{1}, V_{\alpha}^{\prime}\right)$. Then $u=u_{1}+u_{2}$ is the required solution of $T_{\alpha} u=y$.

Consider the linear ordinary differential equation

$$
\alpha \omega \neg \dot{z}-D^{2} H_{0}(z, \quad)=y_{1} .
$$

Let $z: \mathbb{R} \rightarrow V_{\alpha}$ be a solution of this equation, with arbitrary initial condition $z(0)=v_{\alpha} \in V_{\alpha}$. We wish to show that $z(0)=z(2 \pi)$. Let $x \in V_{\alpha}$ and $v \in \operatorname{ker} T_{\alpha}$ satisfy $v(0)=x$. then

$$
\begin{aligned}
0=\int\left\langle y_{1}, v\right\rangle & =\int_{0}^{2 \pi} \alpha \omega(\dot{z}(t), v(t))-D^{2} H_{0}(z(t), v(t)) \mathrm{d} t \\
& =\int_{0}^{2 \pi} \alpha \omega(v(t), z(t))-D^{2} H_{0}(v(t), z(t)) \mathrm{d} t+[\alpha \omega(z(t), v(t))]_{0}^{2 \pi} \\
& =0+\alpha \omega(z(2 \pi)-z(0), x)
\end{aligned}
$$

the integral being 0 since $v \in \operatorname{ker} T_{\alpha}$. Thus $z(2 \pi)=z(0)$ and we may take $u_{1}=z$.

Now consider $\alpha \omega \neg \dot{z}-D^{2} H_{0}(z)=,y_{2}$. The linear map $x \mapsto \omega \neg x$ in this equation is an invertible map $V_{\alpha}^{\prime} \rightarrow\left(V_{\alpha}^{\prime}\right)^{*}$, whose inverse is $J$. The equation becomes

$$
\dot{z}-\alpha^{-1} L z=\alpha^{-1} J y_{2}
$$

defined on $V_{\alpha}^{\prime}$. By writing out the solution explicitly it is easily seen that the unique solution to the initial value problem

$\dot{z}-\alpha^{-1} L z=y_{3} \quad z(0)=[\exp (2 \pi L / \alpha)-I]^{-1} \int_{0}^{2 \pi} \exp (-t L / \alpha) y_{3}(t) \mathrm{d} t$

has period $2 \pi$. The associated map of the circle $\mathbb{R} / 2 \pi \mathbb{Z}$ is then $u_{2}$.

To establish (ii) we continue to use the pairing (3.1) of $X$ and $Y$ but view $X$ as inducing linear functionals on $Y$. Thus we can view $V_{\alpha}$ as $W_{\alpha}^{*}$. Let $x \in X$. By restriction to $W_{\alpha}, x$ induces $\bar{x} \in W_{\alpha}^{*}$, so $\bar{x}=v$ for some $v \in V_{\alpha}$. Thus $x-v$ annihilates $W_{\alpha}$, and hence lies in $Z_{\alpha}$, whence $X=V_{\alpha} \oplus Z_{\alpha}$. (This is the proof given in lemma 2(c) of Magnus (1980).)

We have now engineered ourselves into the precise set-up considered in the splitting lemma of Magnus (1980), there called theorem 1. This states that there exist neighbourhoods $U$ of 0 in $\mathscr{C}^{1}\left(\mathbf{S}^{1}, P\right)$ and $(-\varepsilon, \varepsilon)$ of 0 in $\mathbb{R}$, and a smooth family of diffeomorphisms $\xi_{\tau}: V_{\alpha} \oplus Z_{\alpha} \rightarrow U$ defined on a neighbourhood of $(0,0)$ ) with $\tau \in(-\varepsilon, \varepsilon)$, such that:

(i) $\left.D \xi_{\tau}\right|_{v_{\alpha}}=\mathrm{Id}$,

(ii) $S_{\alpha(1+\tau)}\left(\xi_{\tau}(v, z)\right)=\frac{1}{2}\left\langle\sigma T_{\alpha}(z), z\right\rangle+F_{t}(v)$. 
Here $\langle$,$\rangle is the pairing of X^{*}$ and $X$, and $F_{\tau}(z)$ is a smooth family of smooth functions defined on a neighbourhood of 0 in $V_{\alpha}$. It follows that $\xi_{\tau}(v, z)$ is a critical point of $S_{\alpha(1+\tau)}$ if and only if $z=0$ and $v$ is a critical point of $F_{\tau}$. Thus the problem of finding periodic solutions near 0 , of period near $2 \pi / \alpha$, is reduced to finding critical points of a function defined on a finite-dimensional space.

We now turn to the symmetry properties of $S_{\alpha}$. First note that $S_{\alpha}$ is invariant under the natural phase shift action of $\mathbf{S}^{1} \equiv \mathbb{R} / 2 \pi \mathbb{Z}$ on $\mathscr{C}^{1}\left(\mathbf{S}^{1}, P\right)$ :

$$
(\varphi \cdot u)(\theta)=u(\theta+\varphi) \text {. }
$$

It follows immediately that $R_{\alpha}$ and $T_{\alpha}$ are equivariant with respect to the analogous actions on $X$ and $Y$, and that the decompositions in lemma 3.2 are also invariant under these actions. By the proof of theorem 1 of Magnus (1980), we can choose $\xi_{\tau}$ so that $D \xi_{r}(0,0)$ is the identity. Then $\xi_{\tau}$ can be made $\mathbf{S}^{1}$-equivariant by averaging, and $F_{\tau}(v)$ becomes $\mathbf{S}^{1}$-invariant. For the equivariant splitting lemma in finite dimensions see Arnold (1976).

Identical remarks apply if $\Gamma$ is a compact Lie group acting symplectically on $P$, leaving $H$ invariant, and preserving 0 , the symplectic form $\omega$, and the 1 -form $\beta$. The actions of $\Gamma$ on $X$ and $Y$ are induced from the linear action on $T_{0} P$. The reduced functions $F_{\tau}(v)$ are thus invariant under the action of $\Gamma$ on $V_{\alpha}$ obtained by regarding $V_{\alpha}$ as a resonance subspace of $T_{0} P$.

Finally suppose that $\rho: P \rightarrow P$ is an antisymplectic 'time-reversing' involution with $H \circ \rho=H, \rho(0)=0$, and $\rho^{*} \beta=-\beta$. There are induced actions of $\rho$ on $T_{0} P$, $T_{0}^{*} P$, and $V_{\alpha}$; and we define actions on $\mathscr{C}^{1}\left(\mathbf{S}^{1}, P\right), X$, and $Y$ by $(\rho \cdot u)(\theta)=$ $\rho(u(-\theta))$ in each case. Then $S_{\alpha}$ is invariant under $\rho$, and $R_{\alpha}$ and $T_{\alpha}$ are equivariant. Moreover, as before, the splittings in lemma 3.2 are invariant under the respective actions of $\rho$, so we conclude that the diffeomorphisms $\xi_{\tau}$ are $\rho$-equivariant, and the functions $F_{\tau}(v)$ are $\rho$-invariant. As in section 2, we let

$$
\hat{\mathbf{S}}^{1}= \begin{cases}\mathbf{S}^{1} & \text { if } H \text { is not time-reversible } \\ \mathbf{S}^{1} \rtimes \mathbf{Z}_{2}^{\rho}=\mathbf{O}(2) & \text { if } H \text { is time-reversible. }\end{cases}
$$

We summarise the above discussion in the following result.

Theorem 3.3. For fixed $\alpha$ and for sufficiently small $\tau$ there exists a smooth family of $\Gamma \rtimes \hat{\mathbf{S}}^{1}$-equivariant diffeomorphisms $\xi_{\tau}: V_{\alpha} \oplus Z_{\alpha} \rightarrow \mathscr{C}^{1}\left(\mathbf{S}^{1}, P\right)$, defined on a neighbourhood of $(0,0)$ in $V_{\alpha} \oplus Z_{\alpha}$ and with image a neighbourhood $U$ of 0 in $\mathscr{C}^{1}\left(\mathbf{S}^{1}, P\right)$, and a smooth family of $\Gamma \rtimes \hat{\mathbf{S}}^{1}$-invariant functions $F_{\tau}(v)$ on $V_{\alpha}$, such that the critical points of $S_{\alpha(1+\tau)}$ in $U$ are precisely the points $\xi_{\tau}(v, 0)$ where $v$ is a critical point of $F_{\tau}$.

Thus the periodic trajectories of period $2 \pi / \alpha(1+\tau)$ correspond smoothly and bijectively to the critical points of $F_{\tau}$. Moreover, since $\xi_{\tau}$ is $\Gamma \rtimes \hat{\mathbf{S}}^{1}$-equivariant, this correspondence preserves symmetry groups. Here the symmetry group $\Sigma_{u}$ of a periodic trajectory $u$ is defined to be the isotropy subgroup of the corresponding loop in $\mathscr{C}^{1}\left(\mathbf{S}^{1}, P\right)$ under the $\Gamma \rtimes \hat{\mathbf{S}}^{1}$-action on that space, see Montaldi et al (1988), and the symmetry group of a critical point $v$ of $F_{\tau}$ is the isotropy group of $v$ for the corresponding $\Gamma \rtimes \hat{\mathbf{S}}^{1}$-action on $V_{\alpha}$.

The symmetry group of a periodic trajectory contains a considerable amount of information.

(I) The intersection $\Sigma_{u} \cap \mathbf{S}^{1}=\mathbf{Z}_{k}$ for some $k=1,2, \ldots$; and the minimal period of the solution is equal to $2 \pi /[k \alpha(1+\tau)]$. 
(II) The intersection $K=\Sigma_{u} \cap \Gamma$ is the group of spatial symmetries, i.e. elements of $\Gamma$ which fix the trajectory pointwise.

(III) The remaining elements of $\Sigma_{u} \cap\left(\Gamma \rtimes \hat{\mathbf{S}}^{1}\right)$, of the form $(\gamma, \theta)$ where $0 \neq \theta \in \mathbf{S}^{1}$, are spatio-temporal symmetries of $u$ : the element $\gamma$ of $\Gamma$ maps $u$ to itself, phase-shifted by $\theta$. The image of the projection of $\Sigma_{u} \cap\left(\Gamma \times \mathbf{S}^{1}\right)$ into $\mathbf{S}^{1} /\left(\Sigma_{u} \cap \mathbf{S}^{1}\right)$ is $\mathbf{Z}_{k}$ for $k=1,2, \ldots$, or $\mathbf{S}^{1}$. If it is $\mathbb{1}$ or $\mathbf{Z}_{2}$ then $u$ is called a standing wave, if $\mathbf{Z}_{k}$ $k \geqslant 3$ then $u$ is a discrete rotating wave, and if the image is $\mathbf{S}^{1}$ then $u$ is a rotating wave: see Montaldi et al (1988) subsection 4.3.

(IV) In time-reversible systems, a periodic trajectory $u$ for which $\Sigma_{u}$ contains a conjugate of $\mathbf{Z}_{2}^{\rho}$ in $\hat{\mathbf{S}}^{1} \cong \mathbf{O}(2)$ is called a brake orbit. Each brake orbit intersects $\operatorname{Fix}\left(Z_{2}^{\rho}\right)$ in exactly two points, the brake points. For a classical mechanical system ( $q=$ position, $p=$ momentum) these are points at which the velocity vanishes. It is easily seen that if $\mathbf{Z}_{2}^{\rho}$ and $\Gamma$ commute then brake orbits must be standing waves.

In Montaldi et al (1988) we proved an existence theorem for periodic solutions near 0 with periods near $2 \pi / \alpha$. This theorem states that for every isotropy subgroup $\Sigma$ of the $\Gamma \times \mathbf{S}^{1}$-action on $V_{\alpha}$ and for all sufficiently small $\varepsilon$ there exist on each energy level $H=\varepsilon$ at least $\frac{1}{2} \operatorname{dim} \operatorname{Fix}\left(\Sigma ; V_{\alpha}\right)$ periodic trajectories of (3.1) with periods near $2 \pi / \alpha$ and symmetry groups containing $\Sigma$. Note that it is the $\Gamma \times \mathbf{S}^{1}$-action that is to be considered, not that of $\Gamma \rtimes \hat{\mathbf{S}}^{1}$. Our aim in this paper is to obtain more precise information about these nonlinear normal modes by explicitly solving the equation $D F_{\tau}(v)=0$. To do this we first put the Hamiltonian into Birkhoff normal form to 'sufficiently high' degree and then use the fact that for systems in Birkhoff normal form the reductions described above are trivial.

In the next theorem we identify $\operatorname{ker} T_{\alpha}$ with $V_{\alpha}$ by identifying each loop $u$ with its initial point $u(0)$ as described above.

Theorem 3.4. If $H$ is in Birkhoff normal form to degree $k$ then

$$
\frac{1}{2 \pi} j^{k} F_{\tau}(v)=(1+\tau) j^{2} H(v)-j^{k} H(v)
$$

for $v \in V_{\alpha}$.

Proof. First note that $j^{k} F_{\tau}$ depends only on $j^{k} S_{\alpha(1+\tau)}$ and hence only on $j^{k} H$. Thus it suffices to show that if $H$ is in exact Birkhoff normal form then for $v \in V_{\alpha}$

$$
\frac{1}{2 \pi} F_{\tau}(v)=(1+\tau) H_{2}(v)-H(v) .
$$

We claim that for $H$ in Birkhoff normal form, $F_{\tau}$ is just the restriction of $S_{\alpha(1+\tau)}$ to $V_{\alpha}$. Given this, the result is immediate: for $v \in V_{\alpha}=\operatorname{ker} T_{\alpha}$,

$$
\begin{array}{r}
F_{\tau}(v)=S_{\alpha(1+\tau)}(v)=\alpha(1+\tau) \int_{\mathbf{S}^{1}} v^{*} \beta-\int_{0}^{2 \pi} H \circ v(\theta) \mathrm{d} \theta \\
=(1+\tau) \int_{0}^{2 \pi} H_{2}(v(\theta)) \mathrm{d} \theta-\int_{0}^{2 \pi} H(v(\theta)) \mathrm{d} \theta
\end{array}
$$

which, by $\mathbf{S}^{1}$-equivariance of $H$, is just $2 \pi\left[(1+\tau) H_{2}(v)-H(v)\right]$ as required.

The claim itself follows from the equivariance of $R_{o:(1+\tau)}$ under the $\mathbf{T}^{r} \times \mathbf{S}^{1}$ actions on $X$ and $Y$. By lemma 3.5 below, $V_{\alpha}=\operatorname{Fix}(G(\alpha) ; X)$ and $W_{\alpha}=$ $\operatorname{Fix}(G(\alpha) ; Y)$, whence $R_{\alpha(1+\tau)}\left(V_{\alpha}\right) \subset W_{\alpha}$. It follows that $D S_{\alpha(1+\tau)}(v) \in \sigma\left(W_{\alpha}\right)$ annihilates $\mathbf{Z} \alpha$ for all $v \in V_{\alpha}$. This is precisely the condition given in the last sentence of the splitting lemma of Magnus (1980), under which $F$ is the restriction of $S_{\alpha(1+\tau)}$ to $V_{\alpha}$. 
Lemma 3.5. If $H$ is in exact Birkhoff normal form, that is, is invariant under $\mathbf{T}^{r}$, then $S_{\alpha}$ is $\mathbf{T}^{r} \times \mathbf{S}^{1}$-invariant, and, moreover, for each $\alpha$ there exists a subgroup $G(\alpha) \subset \mathbf{T}^{r} \times \mathbf{S}^{1}$ such that $\operatorname{ker} T_{\alpha}=\operatorname{Fix}(G(\alpha) ; X)$.

Proof. As in section 2, let the decomposition of $V$ into resonance spaces be

$$
V=\bigoplus_{j=1}^{r} V_{\alpha_{j}}
$$

Suppose without loss of generality that $\alpha / \alpha_{1}=k \in \mathbb{N}$, and let $\mathbf{T}^{r-1}=\mathbf{S}_{2} \times \ldots \times \mathbf{S}_{r} \subset$ $\mathbf{T}^{r}$. Then Fix $\left(\mathbf{T}^{r-1} ; X\right)=\mathscr{C}^{1}\left(\mathbf{S}^{1}, V_{\alpha_{1}}\right)=X_{1}$. Now $X_{1}$ carries an $\mathbf{S}^{1} \times \mathbf{S}^{1}$-action, and $V_{\alpha}=\operatorname{ker} T_{\alpha}=\operatorname{Fix}\left(\left(\mathbf{S}^{1} \times \mathbf{Z}^{k}\right)^{-} ; X_{1}\right)$, where

Thus

$$
\left(\mathbf{S}^{1} \times \mathbf{Z}^{k}\right)^{\sim}=\left\{(\theta, \varphi) \in \mathbf{S}^{1} \times \mathbf{S}^{1}: \varphi+\theta \in \mathbf{Z}_{k}\right\}
$$

$$
G(\alpha)=\mathbf{T}^{r-1} \times\left(\mathbf{S}^{1} \times \mathbf{Z}^{k}\right)^{\sim}
$$

If we define $H_{\tau, \alpha}=\left.\left(H-(1+\tau) H_{2}\right)\right|_{V_{\alpha}}$ then theorem 3.4 says that $F(v, \tau)=$ $-2 \pi H_{\tau, \alpha}(v)+R(v, \tau)$ where $R(v, \tau)=\mathrm{O}\left(|v|^{k+2}\right)$. If $H$ is in exact Birkhoff normal form, then $F(v, \tau)=-2 \pi H_{\tau, \alpha}(v)$ and the $\mathbf{S}^{1}$ orbits of critical points of $H_{\tau, \alpha}$ are the relative equilibria of the restricted Hamiltonian system $\left.H\right|_{v_{\alpha}}$, with respect to the $\mathbf{S}^{1}$-action; see, for example, Churchill et al (1983).

\section{Hamiltonian systems with $D_{n}$ symmetry}

We now show how the previous two sections can be used to give precise results on the existence and non-existence of nonlinear normal modes of Hamiltonian systems with dihedral group symmetry. In subsection 4.1 we treat the general case and in subsection 4.2 we discuss the effects of time-reversal symmetry. The stability of these nonlinear normal modes is discussed in Montaldi et al (1988), which also applies the results to Hamiltonians of the 'kinetic energy plus potential energy' type.

\subsection{The general case}

Let $H$ be a Hamiltonian which is invariant under an action of $\mathbf{D}_{n}$ on the phase space $P$ and let $p$ be an equilibrium point of $H$ which is fixed by $\mathbf{D}_{n}$. Throughout this section we assume that $n \geqslant 3$. Suppose that $J D^{2} H(p)$ has a conjugate pair of imaginary eigenvalues $\pm i \alpha$. The flow of the linearised equation (2.2) at $p$ defines an action of $\mathbf{S}^{1}$ on the resonance subspace $V_{\alpha}$ which commutes with that of $\mathbf{D}_{n}$. Generically $V_{\alpha}$ is an irreducible representation of $\mathbf{D}_{n} \times \mathbf{S}^{1}$, and hence is either twoor four-dimensional. By Liapunov's theorem the two-dimensional subspaces yield single families of nonlinear normal modes. Here we consider four-dimensional subspaces, identified with $\mathbb{C}^{2}$, on which $\mathbf{D}_{n}$ acts by

$$
\begin{array}{ll}
\theta \cdot\left(z_{1}, z_{2}\right)=\left(\mathrm{e}^{\mathrm{i} \theta} z_{1}, \mathrm{e}^{-\mathrm{i} \theta} z_{2}\right) & \theta=\frac{2 \pi r}{n} r=0, \ldots, n-1 \\
\kappa \cdot\left(z_{1}, z_{2}\right)=\left(z_{2}, z_{1}\right) &
\end{array}
$$


and $\mathbf{S}^{1}$ by

$$
\varphi \cdot\left(z_{1}, z_{2}\right)=\left(\mathrm{e}^{\mathrm{i} \varphi} z_{1}, \mathrm{e}^{\mathrm{i} \varphi} z_{2}\right) \quad \varphi \in \mathbb{R} / 2 \pi \mathbb{Z} .
$$

Two remarks on this choice of action are in order.

(I) Suppose $\mathbf{D}_{n}$ acts on the 'configuration space' $\mathbb{R}^{2}$ in the usual way (with $\left.\boldsymbol{K} \cdot\left(q_{1}, q_{2}\right)=\left(q_{1},-q_{2}\right)\right)$ and by the complexified action on the 'phase space' $\mathbb{C}^{2}$ with coordinates $w_{j}=(1 / \sqrt{2})\left(p_{j}+\mathrm{i} q_{j}\right)$. As usual we let $\mathbf{S}^{1}$ act on $\mathbb{C}^{2}$ by

$$
\varphi \cdot\left(w_{1}, w_{2}\right)=\left(\mathrm{e}^{\mathrm{i} \varphi} w_{1}, \mathrm{e}^{\mathrm{i} \varphi} w_{2}\right)
$$

Then the action of $\mathbf{D}_{n} \times \mathbf{S}^{1}$ defined by (4.1) is obtained by the change of coordinates $z_{1}=w_{1}+\mathrm{i} w_{2}, z_{2}=w_{1}-\mathrm{i} w_{2}$.

(II) The discussion of nonlinear normal modes coming from other fourdimensional irreducible representations of $\mathbf{D}_{n} \times \mathbf{S}^{1}$ can be reduced to this one by a process similar to that described for $\Gamma=\mathbf{O}(2)$ in example 7.2 of Montaldi et al (1988).

The possible symmetry groups of nonlinear normal modes near normal modes in $V_{\alpha}$ are found by calculating the isotropy subgroups of the action of $\mathbf{D}_{n} \times \mathbf{S}^{1}$ given by $(4.1)$.

Proposition 4.1. The isotropy subgroups of the action of $\mathbf{D}_{n} \times \mathbf{S}^{1}$ defined by (4.1) are conjugate to those listed in table 1.

Proof. See Golubitsky and Stewart (1986) p 136 or Golubitsky et al (1988) pp 368-9. Some small changes have been made to the notation. In particular, the superscripts on $\mathbf{Z}_{2}$ denote generators: $K \in \mathbf{D}_{n}, K^{\prime}=\kappa^{\circ}(-2 \pi / n) \in \mathbf{D}_{n}, \quad(\kappa, \pi) \in$ $\mathbf{D}_{n} \times \mathbf{S}^{1}$ and $(\pi, \pi) \in \mathbf{D}_{n} \times \mathbf{S}^{1}$. The group of order $n$ generated by $(2 \pi / n,-2 \pi / n)$ $\in \mathbf{D}_{n} \times \mathbf{S}^{\mathbf{l}}$ is denoted $\tilde{\mathbf{Z}}_{n}$.

Associated with every periodic trajectory $u(t)$ are other periodic trajectories $\gamma u(t)$ in the same $\Gamma$-orbit. Two such trajectories determine distinct point sets if one is not a phase-shifted version of the other. The number of such point sets is given by the index in $\Gamma$ of the projection of $\Sigma$ into $\Gamma$; see Golubitsky and Stewart (1985), remark 1.3. The second column in table 1 lists this number.

Table 1. Representatives of conjugacy classes of proper isotropy subgroups of the action of $\mathbf{D}_{n} \times \mathbf{S}^{1}$ on $\mathbb{C}^{2}$ given by (4.1).

\begin{tabular}{llll}
\hline $\begin{array}{l}\text { Isotropy } \\
\text { subgroup }\end{array}$ & $\begin{array}{l}\text { Number of nonlinear } \\
\text { normal modes } \\
\text { in } \mathbf{D}_{n} \text {-orbit }\end{array}$ & Fix $(\Sigma)$ & Dimension of Fix $(\Sigma)$ \\
\hline$(a) n$ odd & & & \\
$\tilde{\mathbf{Z}}_{n}$ & 2 & $z_{1}=z, z_{2}=0$ & 2 \\
$\mathbf{Z}_{2}^{k}$ & $n$ & $z_{1}=z_{2}=z$ & 2 \\
$\mathbf{Z}_{2}^{(\kappa, \pi)}$ & $n$ & $z_{1}=-z_{2}=z$ & 2 \\
1 & $2 n$ & $\mathbb{C}^{2}$ & 4 \\
& & & \\
$(b) n$ even & & & 2 \\
$\mathbf{Z}_{n}$ & 2 & $z_{1}=z, z_{2}=0$ & 2 \\
$\mathbf{Z}_{2}^{k} \times \mathbf{Z}_{2}^{(\pi, \pi)}$ & $n / 2$ & $z_{1}=z_{2}=z$ & 2 \\
$\mathbf{Z}_{2}^{\kappa} \times \mathbf{Z}_{2}^{(\pi, \pi)}$ & $n / 2$ & $z_{1}=\mathrm{e}^{2 \pi \mathrm{i} / n} z_{2}=z$ & 2 \\
$\mathbf{Z}_{2}^{(\pi, \pi)}$ & $n$ & $\mathbb{C}^{2}$ & 4 \\
\hline
\end{tabular}


There are three conjugacy classes of maximal isotropy subgroups, each with a two-dimensional fixed-point space, and just one of non-maximal isotropy subgroups. In Montaldi et al (1988) we proved that there always exists a family of nonlinear normal modes with each maximal symmetry type. The theorem below outlines another proof of this, together with a proof that generically these are the only nonlinear normal modes that occur. 'Generically' here means that certain combinations of coefficients in the Birkhoff normal form are non-zero. Before stating the theorem we give the general form of a $\mathbf{D}_{n} \times \mathbf{S}^{1}$-invariant function on $V_{\alpha}$, and hence of the Birkhoff normal form.

Proposition 4.2. Suppose $n \geqslant 3$ and let

$$
m= \begin{cases}n & \text { if } n \text { is odd } \\ n / 2 & \text { if } n \text { is even. }\end{cases}
$$

Then every smooth $\mathbf{D}_{n} \times \mathbf{S}^{1}$-invariant function $h: \mathbb{C}^{2} \rightarrow \mathbb{R}$ has the form

$$
h\left(z_{1}, z_{2}\right)=f(N, P, S, T)
$$

where $\left.N=\left|z_{1}\right|^{2}+\left|z_{2}\right|^{2}, P=\left|z_{1}\right|^{2}\left|z_{2}\right|^{2}, S=\left(z_{1} \bar{z}_{2}\right)^{m}+\left(\bar{z}_{1} z_{2}\right)^{m}, T=i\left(\mid z_{1}\right)^{2}-\left|z_{2}\right|^{2}\right)\left[\left(z_{1} \bar{z}_{2}\right)^{m}-\right.$ $\left.\left(\bar{z}_{1} z_{2}\right)^{m}\right]$, and $f$ is a smooth function of $N, P, S$ and $T$.

Proof. See Golubitsky and Stewart (1986) p 139 or Golubitsky et al (1988) p 372 .

The four homogeneous polynomial invariants $N, P, S$ and $T$ generate the ring of $\mathbf{D}_{n} \times \mathbf{S}^{1}$-invariant polynomial functions on $\mathbb{C}^{2}$. They are related by

$$
T^{2}=\left(N^{2}-4 P\right)\left(4 P^{m}-S^{2}\right)
$$

Theorem 2.1 and proposition 4.2 imply that if $H$ is in Birkhoff normal form to degree $k$ then

$$
\left.H\right|_{V_{\alpha}}(z)=\hat{H}(N, P, S, T)+\mathrm{O}\left(|z|^{k+1}\right)
$$

for some smooth function $\hat{H}$. Our main result in this section is the following.

Theorem 4.3. If $H$ is in Birkhoff normal form to degree $k \geqslant 2 m$ and $\left.H\right|_{V_{\alpha}}$ $=\hat{H}+\mathrm{O}\left(|z|^{k+1}\right)$ as in (4.2), then we have the following.

(i) For each maximal isotropy subgroup $\Sigma$ of the action of $\mathbf{D}_{n} \times \mathbf{S}^{1}$ on $V_{\alpha}$ there exists a smooth family of nonlinear normal modes of $H$ with periods near $2 \pi / \alpha$ and symmetry groups equal to $\Sigma$.

(ii) If $(\partial \hat{H} / \partial S)(0) \neq 0$ and $(\partial \hat{H} / \partial P)(0) \neq 0$ for $n \neq 4, \quad(\partial \hat{H} / \partial P)(0) \pm$ $2(\partial \hat{H} / \partial S)(0) \neq 0$ for $n=4$, then these are the only nonlinear normal modes of $H$ with periods near $2 \pi / \alpha$.

Proof. Without loss of generality we may assume $\alpha=1$ and put $V_{\alpha}=V$. By corollary 3.2(ii) the nonlinear normal modes of $H$ near the normal modes in $V$ are precisely the solutions of an equation

$$
D_{\bar{z}} F(z, \tau)=0
$$

where $F: V \times \mathbb{R} \rightarrow \mathbb{R}$ is a smooth $\mathbf{D}_{n} \times \mathbf{S}^{1}$-invariant function. By proposition 4.2, $F(z, \tau)=\hat{F}(N, P, S, T, \tau)$ and if $\left.H\right|_{V_{\alpha}}=\hat{H}+O\left(|z|^{k+1}\right)$ as in (4.2) then

$$
\hat{F}=\hat{H}-(1+\tau) H_{2}+\mathrm{O}\left(|z|^{k+1}\right)
$$


where $H_{2}$ is the quadratic part of $H$. The assumption $\alpha=1$ and the $\mathbf{D}_{n} \times \mathbf{S}^{1}$. invariance imply that $\mathrm{H}_{2}=\mathrm{N}$.

Substituting $\hat{F}$ for $F$ in (4.3) gives

$$
\begin{aligned}
0=\frac{\partial \hat{F}}{\partial N}\left(\begin{array}{l}
z_{1} \\
z_{2}
\end{array}\right)+ & \frac{\partial \hat{F}}{\partial P}\left(\begin{array}{c}
\left|z_{2}\right|^{2} z_{1} \\
\left|z_{1}\right|^{2} z_{2}
\end{array}\right)+m \frac{\partial \hat{F}}{\partial S}\left(\begin{array}{l}
\bar{z}_{1}^{m-1} z_{2}^{m} \\
z_{1}^{m} \bar{z}_{2}^{m-1}
\end{array}\right) \\
& +\mathrm{i} \frac{\partial \hat{F}}{\partial T}\left\{m\left(\left|z_{1}\right|^{2}-\left|z_{2}\right|^{2}\right)\left(\begin{array}{l}
\bar{z}_{1}^{m-1} z_{2}^{m} \\
z_{1}^{m} \bar{z}_{2}^{m-1}
\end{array}\right)+\left[\left(z_{1} \bar{z}_{2}\right)^{m}-\left(\bar{z}_{1} z_{2}\right)^{m}\right]\left(\begin{array}{c}
z_{1} \\
-z_{2}
\end{array}\right)\right\} .
\end{aligned}
$$

To prove (i) we consider each conjugacy class of maximal isotropy subgroups in turn. To find solutions with $\tilde{\mathbf{Z}}_{n}$ symmetry, restrict (4.5) to the subspace $z_{1}=z, z_{2}=0$ to get

$$
0=\frac{\partial \hat{F}}{\partial N}(z, 0, \tau) \cdot z
$$

Using 4.4 we see that solutions with $z \neq 0$ are given by

$$
0=\frac{\partial \hat{H}}{\partial N}(z, 0)-(1+\tau)+R(z, \tau)
$$

where $R(z, \tau)=\mathrm{O}\left(|z|^{k}\right)$. By the implicit function theorem there exists a family of solutions $(z, 0, \tau(z))$ where

$$
\tau(z)=\frac{\partial \hat{H}}{\partial N}(z, 0)-1+\mathrm{O}\left(|z|^{k}\right) .
$$

The calculations for the other maximal symmetry types are similar.

To prove (ii) we use polar coordinates, $z_{j}=r_{j} \mathrm{e}^{\mathrm{i} \theta_{j}}$ in (4.5). We may assume $r_{1} \neq 0$, $r_{2} \neq 0$. Multiply the first component by $\bar{z}_{1}$ and divide by $r_{1}^{2}$, and do the same for the second component with $\bar{z}_{2}$ and $r_{2}^{2}$. Then substract the second component from the first to obtain

$$
\begin{aligned}
0=\frac{\partial \hat{F}}{\partial P}\left(r_{2}^{2}-\right. & \left.r_{1}^{2}\right)-m \frac{\partial \hat{F}}{\partial S}\left(r_{1} r_{2}\right)^{m-2}\left\{r_{1}^{2} \exp \left[\operatorname{im}\left(\theta_{1}-\theta_{2}\right)\right]-r_{2}^{2} \exp \left[-\mathrm{i} m\left(\theta_{1}-\theta_{2}\right)\right]\right\} \\
& +\mathrm{i} \frac{\partial \hat{F}}{\partial T}\left[-m\left(r_{1}^{2}-r_{2}^{2}\right)\left(r_{1} r_{2}\right)^{m-2}\left\{r_{1}^{2} \exp \left[\mathrm{i} m\left(\theta_{1}-\theta_{2}\right)\right]\right.\right. \\
& \left.\left.+r_{2}^{2} \exp \left[-\mathrm{i} m\left(\theta_{1}-\theta_{2}\right)\right]\right\}+4 \mathrm{i}\left(r_{1} r_{2}\right)^{m} \sin \left[m\left(\theta_{1}-\theta_{2}\right)\right]\right] .
\end{aligned}
$$

Take the imaginary part of this equation and divide by $\left(r_{1}^{2}+r_{2}^{2}\right)\left(r_{1} r_{2}\right)^{m-2}$ to get

$$
0=\frac{\partial \hat{F}}{\partial S} \cdot \sin \left[m\left(\theta_{1}-\theta_{2}\right)\right]-\frac{\partial \hat{F}}{\partial T}\left(r_{1}^{2}-r_{2}^{2}\right) \cos \left[m\left(\theta_{1}-\theta_{2}\right)\right] \text {. }
$$

If $(\partial \hat{F} / \partial S)(0) \neq 0$ then on any family of solutions converging to $0, \sin \left[m\left(\theta_{1}-\theta_{2}\right)\right]$ must also converge to 0 .

Now take the real part of (4.6), use (4.7) to introduce the factor $\left(r_{1}^{2}-r_{2}^{2}\right)$ into the term that does not already possess it and then divide by this factor to obtain

$$
0=\frac{\partial \hat{F}}{\partial P}+m \frac{\partial \hat{F}}{\partial S}\left(r_{1} r_{2}\right)^{m-2} \cos \left[m\left(\theta_{1}-\theta_{2}\right)\right]+\mathrm{O}\left(\left|r_{j}\right|^{2 m-2}\right) .
$$

If $m>2$ and $(\partial \hat{F} / \partial P)(0) \neq 0$ then this equation has no solutions near 0 . For $m=2$ (i.e. $n=4)$, if $(\partial \hat{F} / \partial P)(0) \pm 2(\partial \hat{F} / \partial S)(0) \neq 0$ then there can be no solutions near 0 with $\cos \left[m\left(\theta_{1}-\theta_{2}\right)\right] \rightarrow \pm 1$, and so none with $\sin \left[m\left(\theta_{1}-\theta_{2}\right)\right] \rightarrow 0$. It follows that 
there can be no solutions at all with non-maximal symmetry. The result now follows by identifying the relevant coefficients in $\hat{F}$ with those in $\hat{H}$ using (4.4).

4.2. Time-reversible systems

We now suppose that $H$ possesses a time-reversing symmetry $\rho$ which acts on $V_{\alpha} \cong \mathbb{C}^{2}$ by

$$
\rho \cdot\left(z_{1}, z_{2}\right)=\left(-\bar{z}_{2},-\bar{z}_{1}\right)
$$

This choice of action corresponds to the usual time-reversal operation $(p, q) \mapsto$ $(-p, q)$ under the change of coordinates described in the remark following (4.1). The action of $\mathbf{D}_{n}$ commutes with that of $\rho$ and combined with that of $\mathbf{S}^{1}$ defines an action of $\mathbf{D}_{n} \times \mathbf{O}(2)$. The Birkhoff normal form of $H$ is invariant under this action.

Proposition 4.4. If $n \geqslant 3$ then every smooth $\mathbf{D}_{n} \times \mathbf{O}(2)$-invariant function $h: \mathbb{C}^{2} \rightarrow \mathbb{R}$ has the form

$$
h\left(z_{1}, z_{2}\right)=f(N, P, S)
$$

where $N, P$ and $S$ are as in proposition 4.2 and $f$ is smooth.

Proof. By proposition 4.2 and the relation $T^{2}=\left(N^{2}-4 P\right)\left(4 P^{m}-S^{2}\right)$, every $\mathbf{D}_{n} \times \mathbf{S}^{1}$-invariant function can be written uniquely as

$$
h\left(z_{1}, z_{2}\right)=f_{\text {even }}(N, P, S)+f_{\text {odd }}(N, P, S) \cdot T .
$$

Since $T(\rho z)=-T(z)$ this means that $h$ is invariant under $\rho$ if and only if $f_{\text {odd }} \equiv 0$.

In addition to simplifying the Birkhoff normal forms, time-reversal symmetry also increases the number of possible symmetry types of nonlinear normal modes. These are given by the isotropy subgroups of the $\mathbf{D}_{n} \times \mathbf{O}(2)$-action on $\mathbb{C}^{2}$.

Proposition 4.5. When $n \geqslant 3$ the isotropy subgroups of the action of $\mathbf{D}_{n} \times \mathbf{O}(2)$ on $\mathbb{C}^{2}$ given by (4.1) and (4.8) are conjugate to those shown in figure 1 , which also indicates the partial ordering of conjugacy classes induced by inclusion of isotropy subgroups.

Remark. As before, there are three maximal symmetry types. However, each has gained an extra symmetry involving time-reversal-the standing waves have become brake orbits and the discrete rotating waves are invariant under simultaneous spatial reflection and time reversal. In addition there are several non-maximal symmetry types, including brake orbits which are not aligned along or perpendicular to a polygonal symmetry line, and loop orbits which are symmetric about these lines. The different types are illustrated schematically in figure 2 .

Proof of proposition 4.5. Every isotropy subgroup of the action of $\mathbf{D}_{n} \times \mathbf{O}(2)$ on $\mathbb{C}^{2}$ is conjugate to one generated by an isotropy subgroup of the $\mathbf{D}_{n} \times \mathbf{S}^{1}$-action together with $(\gamma, \rho)$ for some element $\gamma$ in $\mathbf{D}_{n}$ of order 2. A routine case-by-case analysis yields the result. 

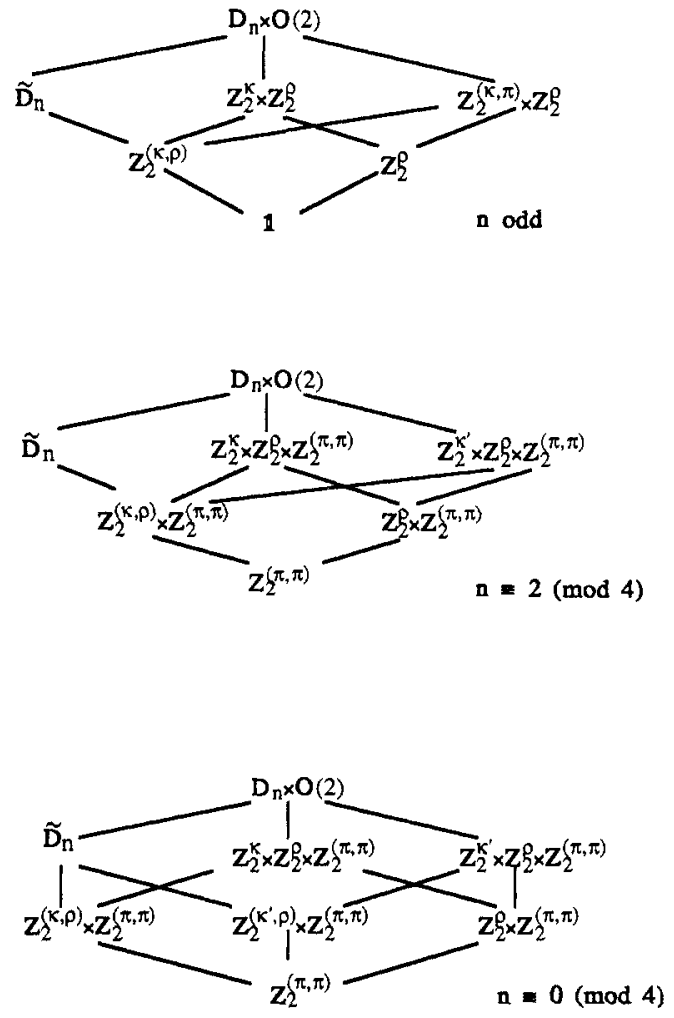

Figure 1. Representatives of the conjugacy classes of isotropy subgroups of the $\mathbf{D}_{n} \times \mathbf{O}(2)$ action on $\mathbb{C}^{2}$ defined by (4.1) and (4.8) when $n \geqslant 3$. The notation is essentially as in table 1 , $\tilde{\mathbf{D}}_{n}$ being the group generated by $\tilde{\mathbf{Z}}_{n}$ and $(\kappa, \rho) \in \mathbf{D}_{n} \times \mathbf{O}(2)$.

Note that the fixed-point spaces are not symplectic (they have dimensions 1 and 3 ) and in fact the periodic orbits do not lie in them: instead they lie in their images under the $\mathbf{S}^{1}$-action.

Finally, we consider the existence of these nonlinear normal modes. In fact, for generic systems the extra time-reversal symmetry has little effect. Theorem 4.3 continues to apply as it stands, and so we again obtain families of nonlinear normal modes for each maximal symmetry type (now with the extra symmetries involving time reversal). If the non-degeneracy conditions $(\partial \hat{H} / \partial S)(0) \neq 0$ and $(\partial \hat{H} / \partial P)(0) \neq 0$ $(n \neq 4), \quad(\partial \hat{H} / \partial P)(0) \pm 2(\partial \hat{H} / \partial S)(0) \neq 0 \quad(n=4)$ hold, then these are the only nonlinear normal modes with periods near $2 \pi / \alpha$. The time-reversal symmetry becomes more interesting if these non-degeneracy conditions are not satisfied, as is possible in generic one-parameter families of $\mathbf{D}_{n}$-symmetric Hamiltonians. Preliminary calculations show that the violation of $(\partial \hat{H} / \partial S)(0) \neq 0$ leads to the appearance of asymmetric brake orbits (isotropy subgroups conjugate to $\mathbf{Z}_{2}^{\rho}$, or $\mathbf{Z}_{2}^{\rho} \times \boldsymbol{Z}_{2}^{(\pi, \pi)}$ ) while for $n \neq 4$ the violation of $(\partial \hat{H} / \partial P)(0) \neq 0$ yields symmetric loops $\left(\boldsymbol{Z}_{2}^{(\kappa, \rho)}\right.$ for $n$ odd, $\mathbf{Z}_{2}^{(\kappa, \rho)} \times \mathbf{Z}_{2}^{(\pi, \pi)}$ for $n \equiv 2(\bmod 4)$ and both $\mathbf{Z}_{2}^{(\kappa, \rho)} \times \mathbf{Z}_{2}^{(\pi, \pi)}$ and $\mathbf{Z}_{2}^{\left(\kappa^{\prime}, \rho\right)} \times \mathbf{Z}_{2}^{(\pi, \pi)}$ for $n \equiv 0(\bmod 4))$. For $n=4$ the degeneracy $(\partial \hat{H} / \partial P)(0)=-2(\partial \hat{H} / \partial S)(0)$ gives nonlinear normal modes with symmetry group $\mathbf{Z}_{2}^{(\kappa, \rho)} \times \mathbf{Z}_{2}^{(\pi, \pi)}$ while the degeneracy $(\partial \hat{H} / \partial P)(0)=2(\partial \hat{H} / \partial S)(0)$ gives nonlinear normal modes with symmetry group $\mathbf{Z}_{2}^{\left(\kappa^{\prime}, \rho\right)} \times \mathbf{Z}_{2}^{(\pi, \pi)}$. 

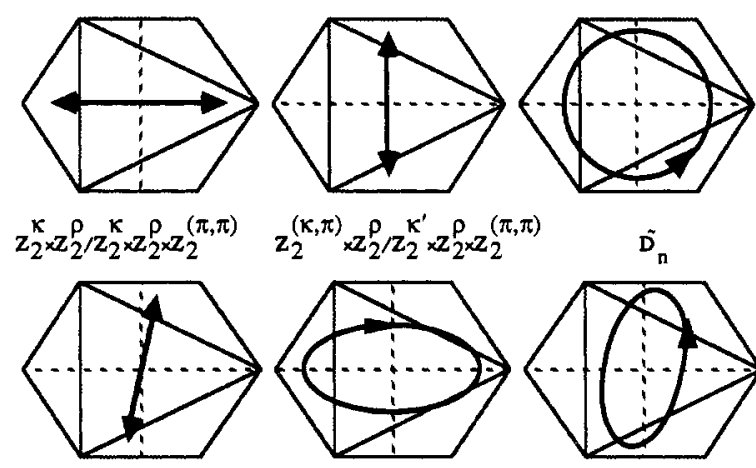

$z_{2}^{(\kappa, \pi)} \times z_{2}^{\rho} / z_{2}^{k^{\prime}} \times z_{2}^{\prime} \times z_{2}^{(\pi, \pi)}$
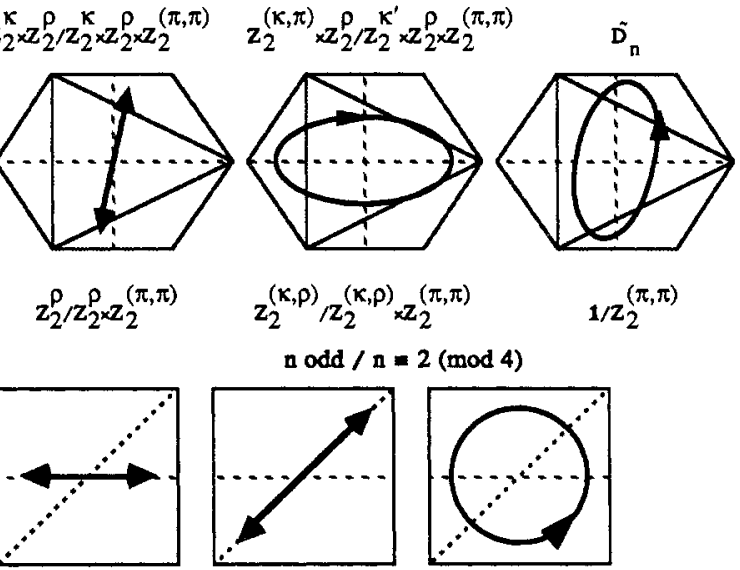

$n$ odd $/ n=2(\bmod 4)$
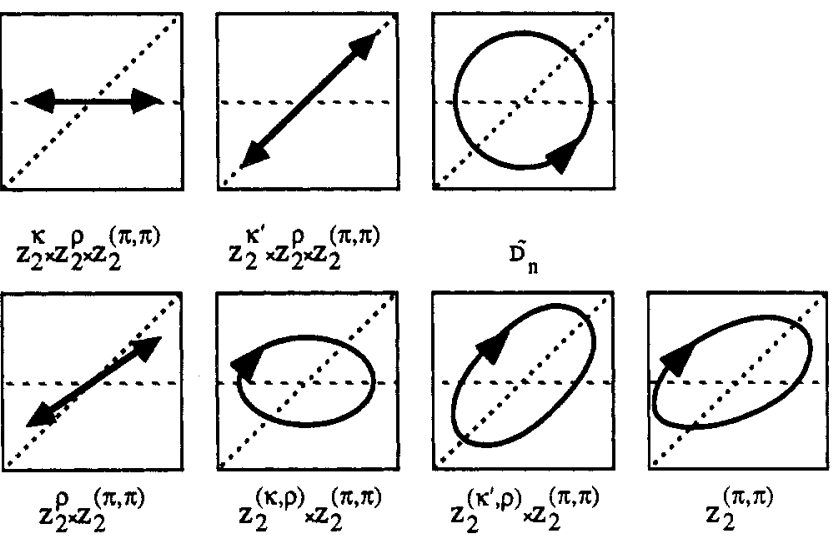

$n=0(\bmod 4)$

Figure 2. Symmetry types of possible nonlinear normal modes in time-reversible $\mathbf{D}_{n}$-symmetric systems. The schematic picture shows the projection of the trajectory into configuration space for a $\mathbf{D}_{n}$-symmetric potential-well Hamiltonian.

\section{Finite determinacy}

In this section we prove a theorem (5.1 below) which gives sufficient conditions on truncated normal forms for them to have precisely the same nonlinear normal modes as the full system. The analogous problem for the stability of nonlinear normal modes is considered in Montaldi et al (1990).

Our theorem is based on recent singularity-theoretic results of Damon (1988). Duistermaat (1984) and van der Meer (1985) have also used singularity theory to treat similar problems, but their approach differs from ours and uses a different context for singularity theory.

Later in this section we shall have to complexify phase space, so we temporarily abandon the complex notation, and work on $\mathbb{R}^{2 n}$ with the symplectic involution $J=\left(\begin{array}{ll}0 & -I \\ I & 0\end{array}\right)$. Thus Hamilton's equation takes the form

$$
\dot{x}=J D_{x} H(x) \quad x \in \mathbb{R}^{2 n} .
$$


We continue to suppose that $H(0)=0=D_{x} H(0)$ and that $L=J D_{x}^{2} H(0)$ is semisimple, has imaginary eigenvalues and is fully resonant. Without loss of generality we may assume that all the eigenvalues are integer multiples of $\mathrm{i}$. The operator $L$ generates a symplectic action of $\mathbf{S}^{1}$ on $\mathbb{R}^{2 n}$, as described in section 2 . We also suppose there is a symplectic action of $\Gamma$ which leaves $H$ invariant, and allow for the possibility that $H$ is time-reversible, i.e. invariant under the involution $\left(\begin{array}{rr}-I & 0 \\ 0 & I\end{array}\right)$, by defining $\hat{\Gamma}$ and $\mathbf{S}^{1}$ as in section 2 . Here the Hamiltonian is invariant under $\hat{G}$ where $G=\Gamma \times \mathbf{S}^{1}$, so $\hat{G}$ can be written as $\Gamma \rtimes \hat{\mathbf{S}}^{1}$. When $\mathbf{Z}_{2}^{\rho}$ commutes with $\Gamma$ and $H$ is time-reversible, as is the case for classical mechanical systems, then $\Gamma \rtimes \hat{\mathbf{S}}^{1} \cong \Gamma \times \hat{\mathbf{S}}^{1} \cong \Gamma \times \mathbf{O}(2)$.

We now state the main theorem. It involves a non-degeneracy condition (NDC1) described in detail below.

Theorem 5.1. Assume that $H(0)=0=D_{x} H(0)$ and that $L=J D_{x}^{2}(H(0)$ is semisimple, has imaginary eigenvalues and is fully resonant. Suppose that $H=H_{2}+H_{k}+\tilde{H}$, where $H_{k}$ is homogeneous of degree $k, j^{k} \tilde{H}=0$ and the truncated Hamiltonian $\mathrm{H}_{2}+\mathrm{H}_{k}$ is in exact Birkhoff normal form and satisfies the nondegeneracy condition (NDC1) described below. Then there exists a $\hat{\Gamma}$-equivariant homeomorphism germ $\left(\mathbb{R}^{2 n} \times \mathbb{R},(0,0)\right) \rightarrow\left(\mathbb{R}^{2 n} \times \mathbb{R},(0,0)\right)$ which maps the nonlinear normal modes of $\mathrm{H}$ to those of $\mathrm{H}_{2}+\mathrm{H}_{k}$, preserving their symmetry groups.

To make this precise we need to describe the non-degeneracy condition (NDC1) on $\mathrm{H}_{2}+\mathrm{H}_{k}$, and to do that we recall some (equivariant) singularity theory. Let $G$ be a compact Lie group acting linearly on $\mathbb{R}^{m}$ and $\mathbb{R}^{p}$. Two $G$-equivariant map germs $f, g: \mathbb{R}^{m}, 0 \rightarrow \mathbb{R}^{p}, 0$ are said to be $G-\mathscr{K}$-equivalent if there exists a $G$ equivariant diffeomorphism germ $X: \mathbb{R}^{m}, 0 \rightarrow \mathbb{R}^{m}, 0$ and a smooth map germ $S: \mathbb{R}^{m}, 0 \rightarrow \mathbf{G L}(p ; \mathbb{R})$ satisfying

such that

$$
S(\gamma \cdot x)=\gamma \cdot S(x) \cdot \gamma^{-1} \quad \forall \gamma \in G
$$

$$
f(x)=S(x) g(X(x)) .
$$

Note that the zero-sets of $G-\mathscr{K}$-equivalent map germs are $G$-equivariantly diffeomorphic.

The $G$-equivariant map germ $f$ is said to be $G$ - $l$-determined if the condition $j^{l} g=j^{l} f$ implies that $g$ is $G-\mathscr{K}$-equivalent to $f$ for all $G$-equivariant map germs $g$. If $j l f$ is $G$-l-determined then $f$ is equivalent to $j^{l} f$, so the zero-set of $f$ is determined up to equivariant diffeomorphism by its $l$-jet.

Let $H$ be a $\hat{\Gamma}$-invariant Hamiltonian as above, which is in Birkhoff normal form to degree $k$. Let $\varphi=J D_{x} F_{\tau}: \mathbb{R}^{2 n} \times \mathbb{R} \rightarrow \mathbb{R}^{2 n}$ denote the corresponding $\Gamma \times \hat{\mathbf{S}}^{1}$ equivariant mapping obtained from the splitting lemma, as in section 3 . Then

$$
j^{k} \varphi=J D_{x}\left(-\tau H_{2}+\bar{H}\right)
$$

where $\bar{H}=j^{k} H-H_{2}$. In the theorem $\bar{H}=H_{k}$. We would like to apply the above singularity-theoretic ideas to $\varphi$, but unfortunately it turns out that $k$-jets of the form (5.1) are never $\Gamma \rtimes \hat{\mathbf{S}}^{1}$-finitely determined. Duistermaat's approach can be interpreted as changing the equivalence relation from $\mathscr{K}$ to one for which $\varphi$ is finitely determined, although he uses Liapunov-Schmidt reduction rather than the splitting 
lemma. Instead, we replace $\varphi$ by a closely related map germ $\psi: \mathbb{R}^{2 n} \times \mathbb{R} \times \mathbb{R} \rightarrow \mathbb{R}^{2 n}$, whose definition also involves Liapunov-Schmidt reduction.

Proposition 5.2. There exists a smooth $\Gamma \rtimes \hat{\mathbf{S}}^{1}$-equivariant map germ $\psi: \mathbb{R}^{2 n} \times \mathbb{R} \times$ $\mathbb{R} \rightarrow \mathbb{R}^{2 n}$, where $\Gamma \rtimes \hat{\mathbf{S}}^{1}$ acts trivially on the factor $\mathbb{R} \times \mathbb{R}$ in the source, with the following properties:

(i) $\psi^{-1}(0)$ is $\Gamma \rtimes \hat{\mathbf{S}}^{1}$-equivariantly diffeomorphic to $\varphi^{-1}(0) \times\{0\}$;

(ii) if $H$ is in Birkhoff normal form to degree $k$ then

$$
\begin{aligned}
& j^{k} \psi(x, \lambda, \tau)=J D_{x}\left(-\tau H_{2}+\bar{H}\right)+\lambda D_{x} H_{2} \\
& \left(j^{k} \psi\right)^{-1}(0)=\left(j^{k} \varphi\right)^{-1}(0) \times\{0\} .
\end{aligned}
$$

The proof is given below, after we have described how $\psi$ is used.

The non-degeneracy condition required in the theorem is the following:

(NDC1) the $k$-jet $j^{k} \psi$ given by (5.2) with $\bar{H}=H_{k}$ is $\Gamma \rtimes \hat{\mathbf{S}}^{1}-\mathscr{K}$-finitely determined.

For the theorem to be useful we must be able to verify this condition in examples. There are essentially two ways to do this: by 'infinitesimal' algebraic criteria or by 'geometric' criteria, see Roberts (1986). The infinitesimal criterion for a $G$-equivariant map germ $f$ to be $G$ - $\mathscr{K}$-finitely determined is that its $G$ - $\mathscr{K}$ tangent space $T \mathscr{K}_{G}(f)$ should have finite codimension in the space of all $G$-equivariant mappings. It is in principle possible to apply this method in our examples, but it appears to require more computation than the second approach, which we therefore follow. Moreover, the calculations required for a stability analysis form part of the verification of the geometric criterion, see Montaldi et al (1990).

To apply the geometric criterion for finite determinacy to an equivariant map germ $f: \mathbb{R}^{m}, 0 \rightarrow \mathbb{R}^{p}, 0$ we must complexify to obtain $f_{\mathbb{C}}: \mathbb{C}^{m}, 0 \rightarrow \mathbb{C}^{p}, 0$. Therefore $f$ must be analytic. In our application $f$ is $j^{k} \psi$, which is polynomial. The actions of $G$ on $\mathbb{R}^{m}$ and $\mathbb{R}^{p}$ are also complexified and extended to actions of the complexified reductive Lie group $G_{\mathfrak{C}}$. If $\Gamma$ is finite, as we henceforth assume, this just involves extending the complexified action of $\mathbf{S}^{1}$ to an action of $\mathbb{C}^{*}$ (the multiplicative group of non-zero complex numbers) on $\mathbb{C}^{2 n}$. If the eigenvalues of $L$ are $\left\{ \pm p_{j} \mathrm{i}: p_{j} \in Z_{>0}, j=1 \ldots n\right\}$ then, with appropriate coordinates, this becomes

$$
s \cdot\left(z_{-n}, \ldots, z_{-1}, z_{1}, \ldots, z_{n}\right)=\left(s^{-p_{n}} z_{-n} \ldots s^{-p_{1}} z_{-1}, s^{p_{1}} z_{1} \ldots s^{p_{n}} z_{n}\right) .
$$

Note that all invariants of this action, and hence all invariants of the $\Gamma \times \hat{\mathbb{C}}^{*}$-action (where $\hat{\mathbb{C}}^{*}$ is the complexification of $\hat{\mathbf{S}}^{1}$ ), which vanish at 0 , also vanish on the 'bad set'

$$
Z=\left\{z: z_{j}=0 \forall j>0 \text { or } \forall j<0\right\} .
$$

Let $\psi^{(k)}$ denote the complexification of $j^{k} \psi$. Translating the geometric criterion for $G$ - $\mathscr{K}$-finite determinacy given in theorem 2.1 of Roberts (1986), we obtain the following alternative form of (NDC1):

(NDC2) in a neighbourhood of $(0,0,0) \in \mathbb{C}^{2 n} \times \mathbb{C} \times \mathbb{C}$ the $k$-jet $\psi_{\mathbb{C}}^{(k)}$ with $\bar{H}=H_{k}$ is a submersion at all points of $\left(\psi_{c}^{(k)}\right)^{-1}(0) \backslash Z$.

We repeat that $\Gamma$ is assumed finite for condition (NDC2). Indeed, (NDC2) never holds if $\Gamma$ is of dimension greater than zero.

This condition becomes easier to understand if we explore its meaning for real points of $\left(\psi_{\mathbb{C}}^{(k)}\right)^{-1}(0)$, i.e. for zeros of $j^{k} \psi$. By proposition 5.2, these are precisely 
the points $(x, \tau, 0) \in \mathbb{R}^{2 n} \times \mathbb{R} \times \mathbb{R}$ such that $(x, \tau)$ is a zero of $j^{k} \varphi$, i.e. a critical point of $-\tau H_{2}+\bar{H}$. At such a point

$$
D_{x} \psi_{c}^{(k)}=\left(J D_{x}^{2}\left(-\tau H_{2}+\bar{H}\right),-J D_{x} H_{2}, D_{x} H_{2}\right) .
$$

However, provided $D_{x} H_{2} \neq 0, J D_{x} H_{2}$ and $D_{x} H_{2}$ are independent vectors lying in the kernel of $D_{x}^{2}\left(-\tau H_{2}+\bar{H}\right)$, since $H_{2}$ is a conserved quantity for $-\tau H_{2}+\vec{H}$. Therefore $\psi_{C}^{(k)}$ is a submersion if and only if the rank of $D_{x}^{2}\left(-\tau H_{2}+\bar{H}\right)$ is $2 n-2$, its maximum possible. In Montaldi et al (1990) we show that this is equivalent to the $\mathbf{S}^{1}$-orbit through $x$ being a non-degenerate critical orbit for the restriction of $\mathrm{H}_{2}+\bar{H}$ to $\left\{H_{2}=H_{2}(x)\right\}$, and to the residual infinitesimal Floquet operator (see Montaldi et al (1988), section $6 \mathrm{~b}$ ) of the corresponding nonlinear normal mode being non-singular. Since $\Gamma$ is finite, this is equivalent to the eigenvalue 0 having multiplicity 2 .

Proof of proposition 5.2. Define a mapping $\Psi: \mathscr{C}^{1}\left(\mathbf{S}^{1}, V\right) \times \mathbb{R} \times \mathbb{R} \rightarrow \mathscr{C}^{0}\left(\mathbf{S}^{1}, V\right)$ by

$$
\Psi(u, \tau, \lambda)=J D_{x} H(u)-(1+\tau) \frac{\mathrm{d} u}{\mathrm{~d} \theta}+\lambda D_{x} H_{2}(u) .
$$

Then $\Psi(u, \tau, \lambda)=0$ if and only if $u(\theta /(1+\tau))$ is a solution of

$$
\dot{x}=J D_{x} H(x)+\lambda D_{x} H_{2}(x) \text {. }
$$

Adapting an idea from Alexander and Yorke (1978), lemma 6.1, we calculate that along any solution of (5.3)

$$
\begin{aligned}
& \mathrm{d} H / \mathrm{d} t=\left\langle D_{x} H(x), \dot{x}\right\rangle \\
&=\left\langle D_{x} H(x), J D_{x} H(x)\right\rangle+\lambda\left\langle D_{x} H(x), D_{x} H_{2}(x)\right\rangle \\
&=\lambda\left\langle D_{x} H(x), D_{x} H_{2}(x)\right\rangle .
\end{aligned}
$$

Since $\left\langle D_{x} H(x), D_{x} H_{2}(x)\right\rangle$ is non-zero on some neighbourhood of 0 in $\mathbb{R}^{2 n}$, it follows that when $\lambda \neq 0, H$ is strictly increasing or decreasing along trajectories in that neighbourhood. Thus, periodic solutions can occur only when $\lambda=0$, i.e. as solutions of the original Hamiltonian system.

The mapping $\psi: \mathbb{R}^{2 n} \times \mathbb{R} \times \mathbb{R} \rightarrow \mathbb{R}$ is obtained by applying the LiapunovSchmidt reduction to $\Psi$ at $(u, \tau, \lambda)=(0,0,0)$, see Golubitsky et al $(1988)$, chapter XVI. The equivariance of $\psi$ follows from that of $\Psi$. There is a $\Gamma \rtimes \hat{\mathbf{S}}^{1}$-equivariant embedding $\mathbb{R}^{2 n} \times \mathbb{R} \times \mathbb{R} \rightarrow \mathscr{C}^{1}\left(\mathbf{S}^{1}, \mathrm{~V}\right) \times \mathbb{R} \times \mathbb{R}$ which maps $\psi^{-1}(0)$ diffeomorphically to $\Psi^{-1}(0)$, and hence to $\Psi(,, 0)^{-1}(0) \times\{0\}$. Since $\Psi(,, 0)^{-1}(0)$ is the set of nonlinear normal modes of the Hamiltonian system, property (i) follows from theorem 3.3. If $H$ is in Birkhoff normal form to degree $k$, then so is (5.3) to degree $k-1$, and property (ii) follows from Golubitsky et al (1988), chapter XVI, section 10. To obtain the final statement, consider the inner product of (5.2) with $D_{x} H_{2}$, and use the fact $\left\langle J D_{x} \bar{H}, D_{x} H_{2}\right\rangle=0$.

Proof of theorem 5.1. We apply corollary 1 of section 4 of Damon (1988) to the germ at 0 of the polynomial mapping $j^{k} \psi: \mathbb{R}^{2 n} \times \mathbb{R} \times \mathbb{R} \rightarrow \mathbb{R}^{2 n}$, noting that it is weighted homogeneous if we assign weights 1 to the $x$-variables and $k-2$ to $\lambda$ and $\tau$. If the non-degeneracy condition is satisfied we can conclude that there is a $\Gamma \times \hat{\mathbf{S}}^{1}$-equivariant homeomorphism germ $\left(\mathbb{R}^{2 n} \times \mathbb{R} \times \mathbb{R},(0,0,0)\right) \rightarrow\left(\mathbb{R}^{2 n} \times \mathbb{R} \times \mathbb{R}\right.$, $(0,0,0))$ mapping the germ of $\psi^{-1}(0)$ to the germ of $j^{k} \psi^{-1}(0)$. This, combined with proposition 5.2 , proves the theorem. 


\section{The time-reversible $1: 1$ resonance with $Z_{2}$ symmetry}

In this section we apply the methods of sections $2-5$, especially theorem 5.1 , to a particular choice of $\Gamma$-invariant Hamiltonian. We have chosen the $\mathbf{Z}_{2}$-invariant 1:1 resonance as a reasonably simple but non-trivial case. We follow the convention of distinguishing between $1: 1$ and $1:-1$ resonances. In the former $H_{2}$ is positive definite, while in the latter it is indefinite. In both cases, of course, the eigenvalues have multiplicity 2 . We have also imposed time-reversal symmetry, both to illustrate how it affects the calculations, and to lead to a simpler system of equations. The group-theoretic analysis of symmetry-breaking is summarised in proposition 6.2 , and the existence and stability results are given in theorem 6.3.

We apply the results to a specific classical dynamical system: a nonlinear planar spring pendulum. Here we consider the 1:1 resonance, see table 4 and figure 6 . This system has recently been analysed by Kummer (1986), who uses a 'reduction' approach via Birkhoff normal form. We confirm that his results continue to apply if just the 4-jet of the system is in Birkhoff normal form, i.e. the system has been put in Birkhoff normal form up to order 4.

Other $k: l$ resonances, with or without $\mathbf{Z}_{2}$ and time-reversal symmetries, could be treated similarly using an extension of theorem 5.1 to inhomogeneous $H_{k}$. In most cases the extra symmetries make little difference to the results-they force some of the nonlinear normal modes to be brake orbits or symmetric loops and sometimes double their numbers, see Roberts (1988). Otherwise the results are very similar to those of Duistermaat (1984), who treated the non-symmetric resonances. However, for the 1:-1 resonance the $\mathbf{Z}_{2}$ symmetry imposes much stronger restrictions. Not only does it remove a number of terms, making the calculations simpler, but it also prevents the occurrence of the non-semisimple 1: -1 resonance studied by van der Meer (1985). As a result, the $\mathbf{Z}_{2}$-symmetric 1:-1 resonance behaves very much like the $\mathbf{Z}_{2}$-symmetric $1: 1$ resonance, at least as regards periodic orbits. See remark 6.4(ii) for further details.

\subsection{The general case}

We consider Hamiltonians on $\mathbb{C}^{2}$ which are invariant under the action of $\mathbf{Z}_{2}$ given by

$$
\kappa \cdot\left(z_{1}, z_{2}\right)=\left(z_{2}, z_{1}\right)
$$

and which are time reversible, i.e. invariant under

$$
\rho \cdot\left(z_{1}, z_{2}\right)=\left(-\bar{z}_{1},-\bar{z}_{2}\right) \text {. }
$$

We suppose that $L=J D^{2} H(0)$ has double eigenvalues $\pm i$. Because it commutes with the $\mathbf{Z}_{2}$-action, $L$ is necessarily semisimple and so generates the $\mathbf{S}^{1}$-action

$$
\theta \cdot\left(z_{1}, z_{2}\right)=\left(\mathrm{e}^{\mathrm{i} \theta} z_{1}, \mathrm{e}^{\mathrm{i} \theta} z_{2}\right) .
$$

These three group actions combine to give an action of $\mathbf{Z}_{2} \times \mathbf{O}(2)$. A Hamiltonian in exact Birkhoff normal form must be invariant under this action.

Proposition 6.1. The ring of functions on $\mathbb{C}^{2}$ that are invariant under the action of $\mathbf{Z}_{2} \times \mathbf{O}(2)$ given by (6.1)-(6.3) is generated freely by

$$
N=\left|z_{1}\right|^{2}+\left|z_{2}\right|^{2} \quad P=\left|z_{1}\right|^{2}\left|z_{2}\right|^{2} \quad Q=\operatorname{Re}\left(z_{1} \bar{z}_{2}\right) .
$$


Proof. The ring of $\mathbf{S}^{1}$-invariant functions is generated by $\left|z_{1}\right|^{2},\left|z_{2}\right|^{2}, \operatorname{Re}\left(z_{1} \bar{z}_{2}\right)$ and $\operatorname{Im}\left(z_{1} \bar{z}_{2}\right)$, or equivalently by $\left|z_{1}\right|^{2}+\left|z_{2}\right|^{2}, \operatorname{Re}\left(z_{1} \bar{z}_{2}\right), \quad\left|z_{1}\right|^{2}-\left|z_{2}\right|^{2}$ and $\operatorname{Im}\left(z_{1} \bar{z}_{2}\right)$. The first two of these are even with respect to the $\mathbf{Z}_{2}$-action, the second two odd.

It follows that the ring of $\mathbf{Z}_{2} \times \mathbf{S}^{1}$-invariant functions is generated by

$N=\left|z_{1}\right|^{2}+\left|z_{2}\right|^{2} \quad Q=\operatorname{Re}\left(z_{1} \bar{z}_{2}\right), \quad P=\left|z_{1}\right|^{2}\left|z_{2}\right|^{2} \quad\left(\left|z_{1}\right|^{2}-\left|z_{2}\right|^{2}\right) \operatorname{Im}\left(z_{1} \bar{z}_{2}\right)$.

Here $P$ replaces $\left(\left|z_{1}\right|^{2}-\left|z_{2}\right|^{2}\right)^{2}$. Note that $\operatorname{Im}\left(z_{1} \bar{z}_{2}\right)^{2}$ can be omitted since

$$
\operatorname{Re}\left(z_{1} \bar{z}_{2}\right)^{2}+\operatorname{Im}\left(z_{1} \bar{z}_{2}\right)^{2}=\left|z_{1}\right|^{2}\left|z_{2}\right|^{2} .
$$

The first three generators of (6.4) are even with respect to $\rho$, while the fourth is odd. Since

$$
\left[\left(\left|z_{1}\right|^{2}-\left|z_{2}\right|^{2}\right) \operatorname{Im}\left(z_{1} \tilde{z}_{2}\right)\right]^{2}=\left(N^{2}-4 P\right)\left(P-Q^{2}\right),
$$

the ring of $\mathbf{Z}_{2} \times \mathbf{O}(2)$-invariant functions is generated by $N, P$ and $Q$. There are three generators and a three-dimensional quotient space, so the ring must be free.

Remark. If we were not restricting our analysis to time-reversible Hamiltonians we would have to consider the ring of $\mathbf{Z}_{2} \times \mathbf{S}^{1}$-invariant functions which, by the proof above, is generated by $N, P, Q$ and $\left(\left|z_{1}\right|^{2}-\left|z_{2}\right|^{2}\right) \operatorname{Im}\left(z_{1} \bar{z}_{2}\right)$.

Thus the most general quartic Hamiltonian in exact Birkhoff normal form invariant under the action (6.1)-(6.3), and with $H_{2}=N$ is

where

$$
H=H_{2}+H_{4}
$$

$$
\begin{aligned}
& H_{2}=N \\
& H_{4}=\alpha_{1} N^{2}+\alpha_{2} P+\beta_{1} Q^{2}+\beta_{2} N Q .
\end{aligned}
$$

Note that this is

$$
\begin{aligned}
& \mathbf{U}(2) \text {-symmetric if } \alpha_{2}=\beta_{1}=\beta_{2}=0 \\
& \mathbf{O}(2) \text {-symmetric if } \beta_{1}=\beta_{2}=0 \\
& \mathbf{D}_{4} \text {-symmetric if } \beta_{2}=0 .
\end{aligned}
$$

Here the action of $\mathbf{U}(2)$ is the usual one on $\mathbb{C}^{2}$, that of $\mathbf{O}(2)$ is generated by $\boldsymbol{K}$ and

$$
\varphi \cdot\left(z_{1}, z_{2}\right)=\left(\mathrm{e}^{\mathrm{i} \varphi} z_{1}, e^{-\mathrm{i} \varphi} z_{2}\right)
$$

and that of $\mathbf{D}_{4}$ by $K$ and the element $\varphi=\pi / 2 \in \mathbf{O}(2)$.

The next proposition classifies the possible symmetry groups of nonlinear normal modes of $\mathbf{Z}_{2}$-invariant time-reversible Hamiltonians at $1: 1$ resonance.

Proposition 6.2. The isotropy subgroups of the action of $\mathbf{Z}_{2} \times \mathrm{O}(2)$ on $\mathbb{C}^{2}$ defined by (6.1)-(6.3) are conjugate to those shown in figure 3 , which also show inclusions and fixed-point spaces.

The different symmetry types of possible nonlinear normal modes are illustrated schematically in figure 4. 


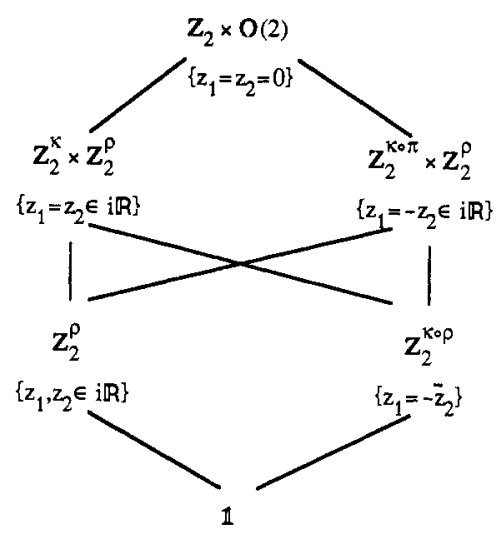

Figure 3. Isotropy lattice for the action of $\mathbf{Z}_{2} \times \mathbf{O}(2)$. The superscripts on $\mathbf{Z}_{2}$ denote generators.

Proof of proposition 6.2. Every $\mathbf{Z}_{2} \times \mathbf{O}(2)$-orbit contains a point with $z_{1}=\mathrm{i} x_{1}$ where $x_{1} \geqslant 0$, so every isotropy subgroup is conjugate to the isotropy subgroup of a point (i $x_{1}, z_{2}$ ) with $x_{1} \geqslant 0$. The only possible elements of such an isotropy subgroup are $\rho, K, K \circ \pi$ and $k \circ \rho$, which easily implies the result.

The main result of this section is the following.

Theorem 6.3. Let $H=H_{2}+H_{4}+\tilde{H}$ be a $\mathbf{Z}_{2}$-invariant time-reversible Hamiltonian with $H_{2}=N, H_{4}=\alpha_{1} N^{2}+\alpha_{2} P+\beta_{1} Q^{2}+\beta_{2} N Q$ and $j^{4} \tilde{H}=0$. Suppose that

$$
\alpha_{2} \neq 0 \quad \beta_{1} \neq 0 \quad \alpha_{2}+\beta_{1} \neq 0 \quad \beta_{1} \pm \beta_{2} \neq 0 \quad \alpha_{2}+\beta_{1} \pm \beta_{2} \neq 0 .
$$

Then the nonlinear normal modes near 0 are as follows.

(i) There is one nonlinear normal mode with symmetry group $\mathbf{Z}_{2}^{K} \times \mathbf{Z}_{2}^{\rho}$. It is stable if $\left(\beta_{1}+\beta_{2}\right)\left(\alpha_{2}+\beta_{1}+\beta_{2}\right)>0$ and unstable if the inequality is reversed.

(ii) There is one nonlinear normal mode with symmetry group $\mathbf{Z}_{2}^{k \circ \pi} \times \mathbf{Z}_{2}^{\rho}$. It is stable if $\left(\beta_{1}-\beta_{2}\right)\left(\alpha_{2}+\beta_{1}-\beta_{2}\right)>0$ and unstable if the inequality is reversed.

(iii) There are two nonlinear normal modes with symmetry group $\mathbf{Z}_{2}^{\rho}$ if $\left|\beta_{2}\right|<\left|\alpha_{2}+\beta_{1}\right|$ and none otherwise. These bifurcate from the $\mathbf{Z}_{2}^{k} \times \mathbf{Z}_{2}^{\rho}$ mode when $\beta_{2}=-\left(\alpha_{2}+\beta_{1}\right)$, and from the $\mathbf{Z}_{2}^{\kappa^{\circ} \pi} \times \mathbf{Z}_{2}^{\rho}$ mode when $\beta_{2}=\left(\alpha_{2}+\beta_{1}\right)$. They are stable if $\alpha_{2}\left(\alpha_{2}+\beta_{1}\right)>0$ and unstable if the inequality is reversed.

(iv) There are two nonlinear normal modes with symmetry group $\mathbf{Z}_{2}^{\text {k॰ } \rho}$ if $\left|\beta_{2}\right|<\left|\beta_{1}\right|$ and none otherwise. These bifurcate from the $\mathbf{Z}_{2}^{\kappa} \times \mathbf{Z}_{2}^{\rho}$ mode when $\beta_{2}=-\beta_{1}$ and from the $\mathbf{Z}_{2}^{\text {Ko }} \times \mathbf{Z}_{2}^{\rho}$ mode when $\beta_{2}=\beta_{1}$. They are stable if $\alpha_{2} \beta_{1}>0$ and unstable if the inequality is reversed.

(v) There are no nonlinear normal modes with trivial symmetry.

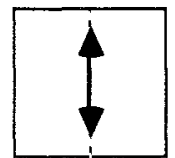

$$
\mathbf{z}_{2}^{\kappa} \times \mathbf{z}_{2}^{\mathrm{p}}
$$

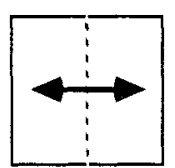

$z_{2}^{K \circ \pi} \times z_{2}^{p}$

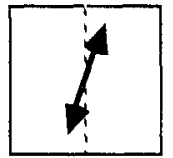

$z_{2}^{\rho}$

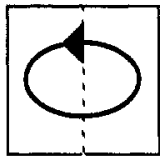

$z_{2}^{K \circ \rho}$

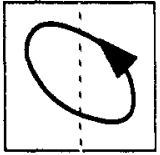

$\mathbb{1}$

Figure 4. Symmetry types of possible nonlinear normal modes. The dotted vertical line is the $\mathbf{Z}_{2}$-symmetry axis. The schematic picture shows the projection of the trajectory into configuration space for a $\mathbf{Z}_{2}$-symmetric potential-well Hamiltonian. 
Proof. The statements about stability of the nonlinear normal modes are proved in Montaldi et al (1990) and are included here for completeness. Here we give a proof of the existence statements, in two parts. In part 1 we find all solutions of $\psi_{C}=0$ where $\psi_{\mathbb{C}}$ is the complex mapping associated to $H_{2}+H_{4}$ in section 5 . From these we pick out the real solutions, and hence find all the nonlinear normal modes of the quartic truncation, together with their bifurcations. In part 2 we check the non-degeneracy condition (NDC2) on each of the solutions of $\psi_{\overparen{C}}=0$, and apply theorem 5.1, to conclude that the result of part 1 for the quartic truncation holds for the full Hamiltonian.

Part 1 of proof. The nonlinear normal modes of the quartic Hamiltonian are given by $\varphi\left(z_{1}, z_{2}, \tau\right)=0$ where $\varphi: \mathbb{C}^{2} \times \mathbb{R} \rightarrow \mathbb{C}^{2}$ is:

$$
\begin{aligned}
& \varphi_{1}\left(z_{1}, z_{2}, \tau\right)=\mathrm{i}\left[\left(-\tau+2 \alpha_{1} N+\alpha_{2}\left|z_{2}\right|^{2}+\beta_{2} Q\right) z_{1}+\left(\frac{1}{2} \beta_{2} N+\beta_{1} Q\right) z_{2}\right] \\
& \varphi_{2}\left(z_{1}, z_{2}, \tau\right)=\mathrm{i}\left[\left(-\tau+2 \alpha_{1} N+\alpha_{2}\left|z_{1}\right|^{2}+\beta_{2} Q\right) z_{2}+\left(\frac{1}{2} \beta_{2} N+\beta_{1} Q\right) z_{1}\right] .
\end{aligned}
$$

The associated mapping $\psi: \mathbb{C}^{2} \times \mathbb{R} \times \mathbb{R} \rightarrow \mathbb{C}^{2}$ is

$$
\psi(z, \tau, \lambda)=\varphi(z, \tau)+\lambda\left(\begin{array}{l}
z_{1} \\
z_{2}
\end{array}\right)
$$

We need to find all zeros of the complexification $\psi_{\mathbb{C}}$ of this mapping, i.e. all solutions of:

$$
\begin{aligned}
& \psi_{1}=\mathrm{i}\left[\left(-\tau+2 \alpha_{1} N+\alpha_{2} z_{2} z_{-2}+\beta_{2} Q\right) z_{1}+\left(\frac{1}{2} \beta_{2} N+\beta_{1} Q\right) z_{2}-\mathrm{i} \lambda z_{1}\right]=0 \\
& \psi_{-1}=-\mathrm{i}\left[\left(-\tau+2 \alpha_{1} N+\alpha_{2} z_{2} z_{-2}+\beta_{2} Q\right) z_{-1}+\left(\frac{1}{2} \beta_{2} N+\beta_{1} Q\right) z_{-2}+\mathrm{i} \lambda z_{-1}\right]=0 \\
& \psi_{2}=\mathrm{i}\left[\left(-\tau+2 \alpha_{1} N+\alpha_{2} z_{1} z_{-1}+\beta_{2} Q\right) z_{2}+\left(\frac{1}{2} \beta_{2} N+\beta_{1} Q\right) z_{1}-\mathrm{i} \lambda z_{2}\right]=0 \\
& \psi_{-2}=-\mathrm{i}\left[\left(-\tau+2 \alpha_{1} N+\alpha_{2} z_{1} z_{-1}+\beta_{2} Q\right) z_{-2}+\left(\frac{1}{2} \beta_{2} N+\beta_{1} Q\right) z_{-1}+\mathrm{i} \lambda z_{-2}\right]=0
\end{aligned}
$$

where now $N=z_{1} z_{-1}+z_{2} z_{-2}, P=z_{1} z_{-1} z_{2} z_{-2}, Q=\frac{1}{2}\left(z_{1} z_{-2}+z_{-1} z_{2}\right)$. Here (6.6a) and (6.6c) are obtained by replacing $\bar{z}_{1}$ and $\bar{z}_{2}$ by $z_{-1}$ and $z_{-2}$ in the two components of $\psi(z, \tau, \lambda)=0$. Equations $(6.6 b)$ and $(6.6 d)$ come from the conjugates of these components. Note that the complexified invariants $N, P$ and $Q$ all vanish on

$$
Z=\left\{\left(z_{1}, z_{-1}, z_{2}, z_{-2}\right): z_{-1}=z_{-2}=0 \text { or } z_{1}=z_{2}=0\right\} \text {. }
$$

To solve the equations (6.6), first note that

$$
(a) \cdot z_{-1}+(b) \cdot z_{1}+(c) \cdot z_{-2}+(d) \cdot z_{2} \Rightarrow \lambda N=0
$$

so either $\lambda=0$ or $N=0$. We consider $N=0$ first. In this case

$$
\text { (a) } \cdot z_{-1}+(c) \cdot z_{-2} \Rightarrow \alpha_{2} P+\beta_{2} Q^{2}=0 \text {. }
$$

Since $\alpha_{2} \neq 0, N=0$ and $\alpha_{2} P+\beta_{2} Q^{2}=0$ define $Z$, together with another branch of solutions with $z_{j} \neq 0, j=1,-1,2,-2$, on which

$$
\tau=\beta_{2} Q \quad \lambda=\frac{\mathrm{i}}{2} \beta_{1} Q \frac{\left(z_{1} z_{-2}-z_{-1} z_{2}\right)}{z_{1} z_{-1}} .
$$

since $N=0$ these solutions are never real.

Now take $\lambda=0$. Then $(a) \cdot z_{-1}+(b) \cdot z_{1}$ implies either

$$
z_{1} z_{-2}-z_{-1} z_{2}=0
$$


or

$$
\frac{1}{2} \beta_{2} N+\beta_{1} Q=0 \text {. }
$$

For $(6.7 a)$ there are three possibilities: (i) solutions in $Z$; (ii) $z_{1}=z_{-1}=0$ or $z_{2}=z_{-2}=0$; (iii) all $z_{j} \neq 0$. We can ignore (i). For (ii), by the symmetry $k$, we need consider only $z_{2}=z_{-2}=0$. The equations (6.6) become

$$
\left(-\tau+2 \alpha_{1} z_{1} z_{-1}\right)\left(\begin{array}{c}
z_{1} \\
z_{-1}
\end{array}\right)=0 \quad \beta_{2} z_{1} z_{-1}\left(\begin{array}{c}
z_{1} \\
z_{-1}
\end{array}\right)=0 .
$$

If $\beta_{2} \neq 0$ the only solutions lie in $Z$. If $\beta_{2}=0$ there is a branch of solutions with $\tau=2 \alpha z_{1} z_{-1}$. These solutions appear again in (iii).

Turning to (iii), if $z_{j} \neq 0$ for all $j$ then by using the $\mathbb{C}^{*}$-action

$$
\sigma \cdot\left(z_{1}, z_{-1}, z_{2}, z_{-2}\right)=\left(\sigma z_{1}, \sigma^{-1} z_{-1}, \sigma z_{2}, \sigma^{-1} z_{-2}\right)
$$

we may assume $z_{-1}=-z_{1}$. Combining this with $z_{1} z_{-2}=z_{-1} z_{2}$ we also have $z_{2}=-z_{-2}$. The equations become

$$
\begin{aligned}
& \left(-\tau+2 \alpha_{1} N-\alpha_{2} z_{2}^{2}+\beta_{2} Q\right) z_{1}+\left(\frac{1}{2} \beta_{2} N+\beta_{1} Q\right) z_{2}=0 \\
& \left(-\tau+2 \alpha_{1} N-\alpha_{2} z_{1}^{2}+\beta_{2} Q\right) z_{2}+\left(\frac{1}{2} \beta_{2} N+\beta_{1} Q\right) z_{1}=0
\end{aligned}
$$

Taking $(6.8 a) \cdot z_{2}-(6.8 b) \cdot z_{1}$ gives

$$
z_{1}^{2}=z_{2}^{2} \quad \text { or } \quad\left(\alpha_{2}+\beta_{1}\right) z_{1} z_{2}+\frac{1}{2} \beta_{2}\left(z_{1}^{2}+z_{2}^{2}\right)=0
$$

The conditions $z_{1}= \pm z_{2}$ give a pair of solution branches:

and

$$
z_{1}=-z_{-1}=z_{2}=-z_{-2} \quad \tau=-\left(4 \alpha_{1}+\alpha_{2}+\beta_{1}+2 \beta_{2}\right) z_{1}^{2}
$$

$$
z_{1}=-z_{-1}=-z_{2}=z_{-2} \quad \tau=-\left(4 \alpha_{1}+\alpha_{2}+\beta_{1}-2 \beta_{2}\right) z_{1}^{2} .
$$

The condition $\left(\alpha_{2}+\beta_{1}\right) z_{1} z_{2}+\frac{1}{2} \beta_{2}\left(z_{1}^{2}+z_{2}^{2}\right)=0$ also gives a pair of solution branches with

$$
\tau=-\left[2 \alpha_{1}\left(z_{1}^{2}+z_{2}^{2}\right)+\beta_{2} z_{1} z_{2}\right]
$$

These bifurcate from the solutions $(6.9 a)$ and $(6.9 b)$ when $\beta_{2}=-\left(\alpha_{2}+\beta_{1}\right)$ and $\beta_{2}=\left(\alpha_{2}+\beta_{1}\right)$, respectively. We recover the solutions of (ii) when $\beta_{2}=0$.

Finally we consider condition $(6.7 b)$, i.e. $\frac{1}{2} \beta_{2} N+\beta_{1} Q=0$. Equations (6.6) become

$$
\begin{aligned}
& \left(-\tau+2 \alpha_{1} N+\alpha_{2} z_{2} z_{-2}+\beta_{2} Q\right)\left(\begin{array}{c}
z_{1} \\
z_{-1}
\end{array}\right)=0 \\
& \left(-\tau+2 \alpha_{1} N+\alpha_{2} z_{1} z_{-1}+\beta_{2} Q\right)\left(\begin{array}{c}
z_{2} \\
z_{-2}
\end{array}\right)=0 .
\end{aligned}
$$

We may assume $\left(z_{1} \neq 0\right.$ or $\left.z_{-1} \neq 0\right)$ and $\left(z_{2} \neq 0\right.$ or $\left.z_{-2} \neq 0\right)$, since otherwise $Q=0$, whence $N=0$. Then

$$
\tau=\left(2 \alpha_{1} N+\alpha_{2} z_{2} z_{-2}+\beta_{2} Q\right)=\left(2 \alpha_{1} N+\alpha_{2} z_{1} z_{-1}+\beta_{2} Q\right)
$$

Since $\alpha_{2} \neq 0$, this implies that $z_{1} z_{-1}=z_{2} z_{-2}$, giving a branch of solutions with

$$
\tau=\left(4 \alpha_{1}+\alpha_{2}-\frac{\beta_{2}^{2}}{\beta_{1}}\right) z_{1} z_{-1} .
$$

These bifurcate from solutions $(6.9 a)$ and $(6.9 b)$ when $\beta_{2}=-\beta_{1}$ and $\beta_{2}=\beta_{1}$, respectively. 
Table 2. Solutions of $\psi_{\mathbb{C}}(z, \tau, \lambda)=0$, assuming $\alpha_{2} \neq 0, \beta_{1} \neq 0, \alpha_{2}+\beta_{1} \neq 0$, and excluding those in $Z$.

\begin{tabular}{llll}
\hline 1 & $z_{1}=-z_{-1}=z_{2}=-z_{-2}$ & $\tau=-\left(4 \alpha_{1}+\alpha_{2}+\beta_{1}+2 \beta_{2}\right) z_{1}^{2}$ & $\lambda=0$ \\
2 & $z_{1}=-z_{-1}=-z_{2}=z_{-2}$ & $\tau=-\left(4 \alpha_{1}+\alpha_{2}-\beta_{1}\right) z_{1}^{2}$ & $\lambda=0$ \\
3 & $z_{1}=-z_{-1}, z_{2}=-z_{-2}$ & $\tau=-\left[2 \alpha_{1}\left(z_{1}^{2}+z_{2}^{2}\right)+\beta_{2} z_{1} z_{2}\right]$ & $\lambda=0$ \\
& $\left(\alpha_{2}+\beta_{1}\right) z_{1} z_{2}+\frac{1}{2} \beta_{2}\left(z_{1}^{2}+z_{2}^{2}\right)=0$ & & \\
4 & $\tau=\left(4 \alpha_{1}+\alpha_{2}-\frac{\beta_{2}^{2}}{\beta_{1}}\right) z_{1} z_{-1}$ & $\lambda=0$ \\
$z_{1} z_{-1}-z_{2} z_{-2}=0$ & & \\
$\frac{1}{2} \beta_{2} N+\beta_{1} Q=0$ & $\tau=\beta_{2} Q$ & $\lambda=\frac{\mathrm{i}}{2} \beta_{1} Q\left(\frac{z_{1} z_{-2}-z_{-1} z_{2}}{z_{1} z_{-1}}\right)$ \\
$5=0$ & & \\
\hline
\end{tabular}

These solutions are summarised in table 2 .

The real solutions are easily extracted from these by putting $z_{-j}=\bar{z}_{j}$ for $j=1,2$. They are summarised in table 3 .

This completes part 1 of the proof of theorem 6.3.

Part 2 of proof. To verify the non-degeneracy condition (NDC2) we must check that $D \psi_{C}$ has rank 4 on each of the solutions in table 2 . We begin with the solutions satisfying $\lambda=0$ - these include the solution of $\hat{\varphi}(z, \tau)=0$. Note that

$$
D \psi=\left(-\frac{1}{2 \pi} \mathrm{i} D_{\bar{z} z}^{2} H_{\tau}+\lambda D_{\bar{z} z}^{2} H_{2},-\mathrm{i} D_{\bar{z}} H_{2}, D_{\bar{z}} H_{2}\right)
$$

and so, when $\lambda=0$,

$$
D \psi=\left(-\frac{1}{2 \pi} \mathrm{i} D_{\bar{z} z}^{2} H_{\tau},-\mathrm{i} D_{\bar{z}} H_{2}, D_{\bar{z}} H_{2}\right) .
$$

Now, $H_{2}$ is a conserved quantity for $H_{\tau}$ and so (for $z \neq 0$ ) $D_{\bar{z}} H_{2}$ and i $D_{\bar{z}} H_{2}$ are independent vectors lying in the null space of $\mathrm{i} D_{\bar{z} z}^{2} H_{\tau}$. Therefore $D \psi$ has rank 4 if and only if $\mathrm{i} D_{\bar{z} z}^{2} H_{\tau}$ has rank 2 . Moreover, the same is true for the complexification.

\begin{tabular}{|c|c|c|c|}
\hline & Symmetry & Solution & Exists \\
\hline 1 & $\mathbf{Z}_{2}^{\kappa} \times \mathbf{Z}_{2}^{\rho}$ & $\begin{array}{l}z_{1}=z_{2}=\mathrm{i} x, x \in \mathbb{R} \\
\tau=\left(4 \alpha_{1}+\alpha_{2}+\beta_{1}+2 \beta_{2}\right) x^{2}\end{array}$ & always \\
\hline 2 & $\mathbf{Z}_{2}^{\kappa \circ \pi} \times \mathbf{Z}_{2}^{\rho}$ & $\begin{array}{l}z_{1}=-z_{2}=\mathrm{i} x, x \in \mathbb{R} \\
\tau=\left(4 \alpha_{1}+\alpha_{2}+\beta_{1}-2 \beta_{2}\right) x^{2}\end{array}$ & always \\
\hline 3 & $\mathbf{z}_{2}^{\rho}$ & $\begin{array}{l}z_{j}=\mathrm{i} x_{j}, x_{j} \in \mathbb{R}(j=1,2) \\
\frac{1}{2} \beta_{2}\left(x_{1}^{2}+x_{2}^{2}\right)+\left(\alpha_{2}+\beta_{1}\right) x_{1} x_{2}=0 \\
\tau=2 \alpha_{1}\left(x_{1}^{2}+x_{2}^{2}\right)+\beta_{2} x_{1} x_{2}\end{array}$ & if $\left|\beta_{2}\right|<\left|\alpha_{2}+\beta_{1}\right|$ \\
\hline 4 & $\mathbf{Z}_{2}^{\kappa \circ \rho}$ & $\begin{array}{l}z_{j}=r \mathrm{e}^{\mathrm{i} \theta_{j}}(j=1,2) \\
\cos \left(\theta_{1}-\theta_{2}\right)=-\beta_{2} / \beta_{1} \\
\tau=\left(4 \alpha_{1}+\alpha_{2}-\beta_{2}^{2} / \beta_{1}\right) r^{2}\end{array}$ & if $\left|\beta_{2}\right|<\left|\beta_{1}\right|$ \\
\hline
\end{tabular}

Table 3. Solutions of $\varphi(z, \tau)=0$, assuming $\alpha_{2} \neq 0, \beta_{1} \neq 0, \alpha_{2}+\beta_{1} \neq 0$. 
Thus it is sufficient to show that $D_{z} \psi_{\mathbb{C}}$ has two non-zero eigenvalues (the maximum possible) on each solution of $\psi_{C}=0$ with $\lambda=0$.

A routine calculation shows $D_{z} \psi_{C}$ to be given by the following partial derivatives:

$$
\begin{aligned}
& \frac{\partial \psi_{1}}{\partial z_{1}}=\mathrm{i}\left[-\tau+4 \alpha_{1} z_{1} z_{-1}+\left(2 \alpha_{1}+\alpha_{2}+\frac{1}{2} \beta_{1}\right) z_{2} z_{-2}+2 \beta_{2} Q\right]-2 \pi \lambda \\
& \frac{\partial \psi_{1}}{\partial z_{-1}}=\mathrm{i}\left[2 \alpha_{1} z_{1}^{2}+\beta_{2} z_{1} z_{2}+\frac{1}{2} \beta_{1} z_{2}^{2}\right] \\
& \frac{\partial \psi_{1}}{\partial z_{2}}=\mathrm{i}\left[\beta_{2} N+\left(2 \alpha_{1}+\alpha_{2}+\frac{1}{2} \beta_{1}\right) z_{1} z_{-2}+\beta_{1} z_{-1} z_{2}\right] \\
& \frac{\partial \psi_{1}}{\partial z_{-2}}=\mathrm{i}\left[\frac{1}{2} \beta_{2} z_{1}^{2}+\left(2 \alpha_{1}+\alpha_{2}+\frac{1}{2} \beta_{1}\right) z_{1} z_{2}+\frac{1}{2} \beta_{2} z_{2}^{2}\right] \\
& \frac{\partial \psi_{-1}}{\partial z_{1}}=-\mathrm{i}\left[2 \alpha_{1} z_{-1}^{2}+\beta_{2} z_{-1} z_{-2}+\frac{1}{2} \beta_{1} z_{-2}^{2}\right] \\
& \frac{\partial \psi_{-1}}{\partial z_{-1}}=-\mathrm{i}\left[-\tau+4 \alpha_{1} z_{1} z_{-1}+\left(2 \alpha_{1}+\alpha_{2}+\frac{1}{2} \beta_{1}\right) z_{2} z_{-2}+2 \beta_{2} Q\right]-2 \pi \lambda \\
& \frac{\partial \psi_{-1}}{\partial z_{2}}=-\mathrm{i}\left[\frac{1}{2} \beta_{2} z_{-1}^{2}+\left(2 \alpha_{1}+\alpha_{2}+\frac{1}{2} \beta_{1}\right) z_{-1} z_{2}+\frac{1}{2} \beta_{2} z_{-2}^{2}\right] \\
& \frac{\partial \psi_{-1}}{\partial z_{-2}}=-\mathrm{i}\left[\beta_{2} N+\beta_{1} z_{1} z_{-2}+\left(2 \alpha_{1}+\alpha_{2}+\frac{1}{2} \beta_{1}\right) z_{-1} z_{2}\right] .
\end{aligned}
$$

The partial derivatives of $\psi_{2}$ and $\psi_{-2}$ are obtained by interchanging the subscripts 1 and 2.

For each solution with $\lambda=0$ we calculate $D_{z} \psi_{\mathbb{C}} \cdot v_{j}$ for an appropriate basis $v_{1}, \ldots, v_{4}$ of $\mathbb{C}^{4}$. In each case we take

Then

$$
v_{1}=\left(\mathrm{i} z_{1},-\mathrm{i} z_{-1}, \mathrm{i} z_{2},-\mathrm{i} z_{-2}\right) \quad v_{2}=\left(z_{1}, z_{-1}, z_{2}, z_{-2}\right) \text {. }
$$

$$
D_{z} \psi_{\mathbb{C}} \cdot v_{1}=0 \quad \text { and } \quad D_{z} \psi_{\mathbb{C}} \cdot v_{2}=\tau v_{1} \text {. }
$$

For the first branch of solutions in table 2 we take

$$
v_{3}=(\mathrm{i} z, \mathrm{i} z,-\mathrm{i} z,-\mathrm{i} z) \quad v_{4}=(z,-z,-z, z) .
$$

Then calculation gives

$$
D_{z} \psi_{\mathbb{C}} \cdot v_{3}=-2\left(\beta_{1}+\beta_{2}\right) z^{2} \cdot v_{4} \quad D_{z} \psi_{C} \cdot v_{4}=2\left(\alpha_{2}+\beta_{1}+\beta_{2}\right) z^{2} \cdot v_{3}
$$

resulting in eigenvalues $\pm 2\left[-\left(\beta_{1}+\beta_{2}\right)\left(\alpha_{2}+\beta_{1}+\beta_{2}\right)\right]^{1 / 2} z^{2}$, which are non-zero if $\beta_{1}+\beta_{2} \neq 0, \alpha_{2}+\beta_{1}+\beta_{2} \neq 0$.

For the second branch of solutions let

$$
v_{3}=(\mathrm{i} z, \mathrm{i} z, \mathrm{i} z, \mathrm{i} z) \quad v_{4}=(z,-z, z,-z) \text {. }
$$

Then calculation yields the same results as for the first branch, but with $\beta_{2}$ replaced by $-\beta_{2}$. Thus $\psi_{\mathbb{C}}$ is non-degenerate along this branch if $\beta_{1}-\beta_{2} \neq 0$ and $\alpha_{2}+\beta_{1}-$ $\beta_{2} \neq 0$.

For the third branch let

$$
v_{3}=\left(\mathrm{i} z_{2}, \mathrm{i} z_{2},-\mathrm{i} z_{1},-\mathrm{i} z_{1}\right) \quad v_{4}=\left(z_{2},-z_{2},-z_{1}, z_{1}\right) .
$$


Then

$D_{2} \psi_{\mathbb{C}} \cdot v_{3}=2 \alpha_{2}\left(z_{1}^{2}+z_{2}^{2}\right) v_{4} \quad D_{2} \psi_{\mathbb{C}} \cdot v_{4}=\left[2 \beta_{2} z_{1} z_{2}+\left(\alpha_{2}+\beta_{1}\right)\left(z_{1}^{2}+z_{2}^{2}\right)\right] v_{3}$

giving eigenvalues

$\pm\left[-\alpha_{2}\left(\alpha_{2}+\beta_{1}\right)\right]^{1 / 2}\left(z_{1}^{2}+z_{2}^{2}\right) \quad$ since $\left(\alpha_{2}+\beta_{1}\right) z_{1} z_{2}+\frac{1}{2} \beta_{2}\left(z_{1}^{2}+z_{2}^{2}\right)=0$.

These are non-zero if $\alpha_{2}+\beta_{1} \pm \beta_{2} \neq 0, \alpha_{2} \neq 0$ and $\alpha_{2}+\beta_{1} \neq 0$.

For the fourth branch let

Then

$$
v_{3}=\left(\mathrm{i} z_{1},-\mathrm{i} z_{-1},-\mathrm{i} z_{2}, \mathrm{i} z_{-2}\right) \quad v_{4}=\left(z_{1}, z_{-1},-z_{2},-z_{-2}\right) .
$$

$$
D_{2} \psi_{\mathbb{C}} \cdot v_{3}=-\left(\beta_{1} N+2 \beta_{2} Q\right) v_{4} \quad D_{2} \psi_{\subset} \cdot v_{4}=-\alpha_{2} N v_{3}
$$

and the eigenvalues are $\pm\left[\alpha_{2} \beta_{1}\left(N^{2}-4 Q^{2}\right)\right]^{1 / 2}$. The expression $N^{2}-4 Q^{2}$ vanishes on this branch only when $\beta_{1} \pm \beta_{2}=0$, and so these eigenvalues are non-zero if $\beta_{1} \pm \beta_{2} \neq 0, \alpha_{2} \neq 0, \beta_{1} \neq 0$.

We now turn to the fifth branch of solutions in table 2 , for which $\lambda \neq 0$. Here we have to consider the whole of $D \psi_{\mathbb{C}}$, including the derivatives with respect to $\tau$ and $\lambda$. We choose the following basis for $\mathbb{C}^{6}=\mathbb{C}^{4} \times \mathbb{C} \times \mathbb{C}$ :

$$
\begin{array}{ll}
v_{1}=\left(\mathrm{i} z_{1},-\mathrm{i} z_{-1}, \mathrm{i} z_{2}, \mathrm{i} z_{-2}, 0,0\right) & v_{2}=\left(z_{1}, z_{-1}, z_{2}, z_{-2}, 0,0\right) \\
v_{3}=\left(\mathrm{i} z_{1},-\mathrm{i} z_{-1},-\mathrm{i} z_{2}, \mathrm{i} z_{-2}, 0,0\right) & v_{4}=\left(z_{1}, z_{-1},-z_{2},-z_{-2}, 0,0\right) \\
v_{5}=(0,0,0,0,1,0) & v_{6}=(0,0,0,0,0,1) .
\end{array}
$$

Then straightforward calculation gives:

$$
\begin{aligned}
D \psi_{\mathbb{C}} \cdot v_{1}= & 0 \quad D \psi_{\mathbb{C}} \cdot v_{2}=2 \tau v_{1} \\
D \psi_{C} \cdot v_{3}= & \mathrm{i} \beta_{2}\left(z_{1} z_{-2}-z_{-1} z_{2}\right) v_{1}+\frac{\beta_{1}}{z_{1} z_{-1}}\left(z_{1}^{2} z_{-2}^{2}+z_{-1}^{2} z_{2}^{2}\right) v_{2}+\frac{\mathrm{i} \beta_{1}}{z_{1} z_{-1}}\left(z_{1}^{2} z_{-2}^{2}+z_{-1}^{2} z_{2}^{2}\right) v_{3} \\
D \psi_{C} \cdot v_{4}= & 4 \alpha_{1}\left(z_{1} z_{-1}-z_{2} z_{-2}\right) v_{1}-\mathrm{i} \beta_{2}\left(z_{1} z_{-2}-z_{-1} z_{2}\right) v_{2}+\beta_{2}\left(z_{1} z_{2}+z_{2} z_{1}\right) v_{3} \\
& +\frac{\mathrm{i} \beta_{1}}{z_{1} z_{-1}}\left(z_{1}^{2} z_{-2}^{2}+z_{-1}^{2} z_{2}^{2}\right) v_{4} \\
D \psi_{C} \cdot v_{5}= & -v_{1} \quad D \psi_{\mathbb{C}} \cdot v_{6}=v_{2} .
\end{aligned}
$$

With respect to the bases $v_{1} \ldots v_{6}$ for $\mathbb{C}^{6}$ and $v_{1} \ldots v_{4}$ for $\mathbb{C}^{4}, D \psi_{\mathbb{C}}$ has the matrix

$$
\left(\begin{array}{rrrrrr}
0 & 2 \tau & * & * & -1 & 0 \\
0 & 0 & * & * & 0 & 1 \\
0 & 0 & \alpha & * & 0 & 0 \\
0 & 0 & 0 & \alpha & 0 & 0
\end{array}\right)
$$

where $\alpha=\left(\mathrm{i} \beta_{1} / z_{1} z_{-1}\right)\left(z_{1}^{2} z_{-2}^{2}-z_{-1}^{2} z_{2}^{2}\right)$ and $*$ denotes an irrelevant entry.

These formulae make sense since $z_{1}=0$ or $z_{-1}=0$ on this branch only at the origin. It follows that $D \psi_{\mathbb{C}}$ has maximal rank if and only if $\alpha \neq 0$, i.e. $\beta_{1} \neq 0$ and $z_{1}^{2} z_{-2}^{2}-z_{-1}^{2} z_{2}^{2} \neq 0$. On these solutions the latter condition is equivalent to $Q \neq 0$ and $z_{1} z_{-2} \neq z_{-1} z_{2}$. The condition $Q \neq 0$ is, in turn, equivalent to $\alpha_{2} \neq 0$ and since $\alpha_{2} P+\beta_{1} Q^{2}=0$, the condition $z_{1} z_{-2} \neq z_{-1} z_{2}$ is equivalent to $\alpha_{2}+\beta_{1} \neq 0$.

This completes the proof of theorem 6.1.

Remark 6.4. (i) We saw in Montaldi et al (1988), remark 1.2 that nonlinear normal modes coming from two-dimensional fixed-point spaces of $\Gamma \times \mathbf{S}^{1}$-actions on 
resonance spaces, such as those with symmetry groups $\mathbf{Z}_{2}^{\kappa} \times \mathbf{Z}_{2}^{\rho}$ and $\mathbf{Z}_{2}^{\kappa \circ \pi} \times \mathbf{Z}_{2}^{\rho}$ in this example, always from smooth manifolds through the origin. It is easily seen from the equations in table 3 that this is also true for the nonlinear normal modes with symmetry groups $\mathbf{Z}_{2}^{\rho}$ and $\mathbf{Z}_{2}^{\mathbf{K}^{\circ} \rho}$ in the truncated normal form. An application of the implicit function theorem extends this result to the full system. The smoothness of these nonlinear normal modes at the origin should be contrasted with the conical singularities that occur in the 1:2 resonance, Duistermaat (1984).

(ii) The computations for the periodic orbits in the $\mathbf{Z}_{2}$-symmetric $1:-1$ resonance are essentially identical to those for the $\mathbf{Z}_{2}$-symmetric 1:1 resonance (in appropriate coordinates). To see this, let $\mathbf{Z}_{2} \times \mathbf{S}^{1}$ act on $\mathbb{C}^{2}$ by

$$
\kappa\left(z_{1}, z_{2}\right)=\left(-z_{1}, z_{2}\right) \quad \theta\left(z_{1}, z_{2}\right)=\left(\mathrm{e}^{\mathrm{i} \theta} z_{1}, \mathrm{e}^{ \pm \mathrm{i} \theta} z_{2}\right)
$$

where the sign \pm corresponds to the $1: \pm 1$ resonance. Time-reversal in both cases acts by

$$
\rho\left(z_{1}, z_{2}\right)=\left(-\bar{z}_{1},-\bar{z}_{2}\right)
$$

The $\mathbf{Z}_{2} \times \mathbf{S}^{1}$-invariants are generated by the following:

$1: 1$ resonance

$$
N_{1}=\left|z_{1}\right|^{2} \quad N_{2}=\left|z_{2}\right|^{2} \quad S=\operatorname{Re}\left(z_{1}^{2} \bar{z}_{2}^{2}\right) \quad T=\operatorname{Im}\left(z_{1}^{2} \bar{z}_{2}^{2}\right)
$$

with the relation $S^{2}+T^{2}=N_{1}^{2} N_{2}^{2}$;

1:- -1 resonance

$$
N_{1}^{\prime}=\left|z_{1}\right|^{2} \quad N_{2}^{\prime}=-\left|z_{2}\right|^{2} \quad S^{\prime}=\operatorname{Re}\left(z_{1}^{2} z_{2}^{2}\right) \quad T^{\prime}=\operatorname{Im}\left(z_{1}^{2} z_{2}^{2}\right)
$$

with the relation $S^{\prime 2}+T^{\prime 2}=N_{1}^{\prime 2} N_{2}^{\prime 2}$.

The identification of $\left(N_{1}, N_{2}, S, T\right)$ and $\left(N_{1}^{\prime}, N_{2}^{\prime}, S^{\prime}, T^{\prime}\right)$ is an isomorphism of Poisson algebras, i.e. $\{S, T\}^{\prime}=\left\{S^{\prime}, T^{\prime}\right\}$, etc. Since all the calculations are algebraic the results are the same, except for the interpretation of which solutions are real. For the 1:1 resonance the reality condition is $N_{1}, N_{2} \geqslant 0$; for the $1:-1$ resonance it is $N_{1}^{\prime} \geqslant 0, N_{2}^{\prime} \leqslant 0$. We leave the remainder of the calculations to the reader.

\subsection{The spring pendulum}

We now apply the above results to the special case of a planar spring pendulum at $1: 1$ resonance, see figure 5. More generally we consider Hamiltonians of the form

$$
H\left(p_{1}, p_{2}, q_{1}, q_{2}\right)=\frac{1}{2}\left(p_{1}^{2}+p_{2}^{2}\right)+V\left(q_{1}, q_{2}\right)
$$

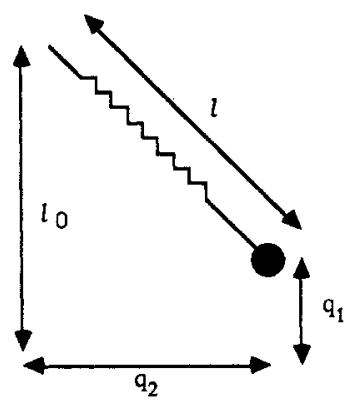

Figure 5. The spring pendulum. 
where $V$ is a smooth potential function on $\mathbb{R}^{2}$, having a non-degenerate minimum at 0 , and invariant under the $\mathbf{Z}_{2}$-action

$$
\boldsymbol{K} \cdot\left(q_{1}, q_{2}\right)=\left(q_{1},-q_{2}\right) .
$$

At $1: 1$ resonance the 4-jet of $V$ is

$j^{4} V\left(q_{1}, q_{2}\right)=\frac{1}{2}\left(q_{1}^{2}+q_{2}^{2}\right)+a_{1} q_{1}^{3}+a_{2} q_{1} q_{2}^{2}+\left(b_{1} q_{1}^{4}+b_{2} q_{1}^{2} q_{2}^{2}+b_{3} q_{2}^{4}\right)$

(assuming, as we may, that $V(0,0)=0$ ). Thus

where

$$
\mathrm{H}=\mathrm{H}_{2}+\mathrm{H}_{3}+\mathrm{H}_{4}+\ldots
$$

$$
\begin{aligned}
& H_{2}=\frac{1}{2}\left(p_{1}^{2}+p_{2}^{2}+q_{1}^{2}+q_{2}^{2}\right) \\
& H_{3}=a_{1} q_{1}^{3}+a_{2} q_{1} q_{2}^{2} \\
& H_{4}=b_{1} q_{1}^{4}+b_{2} q_{1}^{2} q_{2}^{2}+b_{3} q_{2}^{4} .
\end{aligned}
$$

We now define complex coordinates by

$$
z_{1}=\frac{1}{\sqrt{2}}\left(p_{1}+p_{2}+\mathrm{i}\left(q_{1}+q_{2}\right)\right) \quad z_{2}=\frac{1}{\sqrt{2}}\left(p_{1}-p_{2}+\mathrm{i}\left(q_{1}-q_{2}\right)\right) .
$$

Then $K \cdot\left(z_{1}, z_{2}\right)=\left(z_{2}, z_{1}\right)$ as in section 5 , and $H_{2}=N=\left|z_{1}\right|^{2}+\left|z_{2}\right|^{2}$. Therefore the Birkhoff normal form $\hat{H}$ to degree 4 of $H$ has the form (6.5):

where

$$
\hat{H}=\hat{H}_{2}+\hat{H}_{4}
$$

$$
\begin{aligned}
& \hat{H}_{2}=N \\
& \hat{H}_{4}=\alpha_{1} N^{2}+\alpha_{2} P+\beta_{1} N Q+\beta_{2} Q^{2} .
\end{aligned}
$$

We want the coefficients $\alpha_{1}, \alpha_{2}, \beta_{1}, \beta_{2}$ in terms of $a_{1}, a_{2}, b_{1}, b_{2}, b_{3}$. By the general theory, $\hat{H}_{4}$ consists of two terms:

$$
\hat{H}_{4}=\frac{1}{2} \pi\left(\left\{L_{3}, H_{3}\right\}\right)+\pi\left(H_{4}\right) \text {. }
$$

The first of these is calculated in Churchill and Lee (1987) to be

$$
\begin{array}{r}
\frac{1}{2} \pi\left(\left\{L_{3}, H_{3}\right\}\right)=-\frac{5}{4}\left(\frac{3}{4} a_{1}^{2}+\frac{1}{2} a_{1} a_{2}+\frac{5}{12} a_{2}^{2}\right) N^{2}-\left(a_{1} a_{2}-2 a_{2}^{2}\right) P \\
-\frac{1}{4}\left(15 a_{1}^{2}-14 a_{1} a_{2}+3 a_{2}^{2}\right) Q^{2}-\frac{5}{4}\left(3 a_{1}^{2}-\frac{1}{3} a_{2}^{2}\right) N Q .
\end{array}
$$

The second term is obtained by straightforward averaging:

$$
\pi\left(H_{4}\right)=\frac{3}{8}\left(b_{1}+b_{2}+b_{3}\right) N^{2}-b_{2} P+\frac{1}{2}\left(3 b_{1}-b_{2}+3 b_{3}\right) N Q+\frac{3}{2}\left(b_{1}-b_{3}\right) N Q \text {. }
$$

So

$$
\begin{aligned}
& \alpha_{1}=-\frac{5}{4}\left(\frac{3}{4} a_{1}^{2}+\frac{1}{2} a_{1} a_{2}+\frac{5}{12} a_{2}^{2}\right)+\frac{3}{8}\left(b_{1}+b_{2}+b_{3}\right) \\
& \alpha_{2}=-\left(a_{1} a_{2}-2 a_{2}^{2}\right)-b_{2} \\
& \beta_{1}=-\frac{1}{4}\left(15 a_{1}^{2}-14 a_{1} a_{2}+3 a_{2}^{2}\right)+\frac{1}{2}\left(3 b_{1}-b_{2}+3 b_{3}\right) \\
& \beta_{2}=-\frac{5}{4}\left(3 a_{1}^{2}-\frac{1}{3} a_{2}^{2}\right)+\frac{3}{2}\left(b_{1}-b_{3}\right) .
\end{aligned}
$$

Now consider the spring pendulum, as in figure 5. We follow Kummer (1986). Let $l_{0}$ be the length of the spring at the stable equilibrium position $q_{1}=0=q_{2}$, let $m$ be the mass of the pendulum, and $g$ be the acceleration due to gravity. Let $\sigma(l)$ denote the energy stored in the spring at length $l$. For a linear spring, obeying Hooke's Law, $\sigma(l)$ is quadratic; however, we assume a more general form for $\sigma$. The Hamiltonian is

$$
H=\frac{1}{2 m}\left(p_{1}^{2}+p_{2}^{2}\right)+m g q_{1}+\sigma(l)
$$


where $l=\left[\left(l_{0}-q_{1}\right)^{2}+q_{2}^{2}\right]^{1 / 2}$. In fact we may choose units for mass, length and time so that $m=l_{0}=g=1$, in which case

$$
H=\frac{1}{2}\left(p_{1}^{2}+p_{2}^{2}\right)+q_{1}+\sigma(l)
$$

where $l=\left[\left(1-q_{1}\right)^{2}+q_{2}^{2}\right]^{1 / 2}$. Note that we must have $\sigma^{\prime}(1)=1$ for the equilibrium to be at $q_{1}=0=q_{2}$.

Let $c=\sigma^{\prime \prime}(1), d=\sigma^{\prime \prime \prime}(1)$ and $f=\sigma^{\mathrm{iv}}(1)$. For a linear spring, $c$ is equal to the spring constant and all higher-order coefficients are zero. The Taylor expansion of $H$ to degree 4 is

where

$$
H=H_{2}+H_{3}+H_{4}+\ldots
$$

$$
\begin{aligned}
& H_{2}=\frac{1}{2}\left(p_{1}^{2}+p_{2}^{2}+\omega^{2} q_{1}^{2}+q_{2}^{2}\right) \\
& H_{3}=-\frac{1}{6} d q_{1}^{3} \\
& H_{4}=\frac{1}{24} f q_{1}^{4}+\frac{1}{4} d q_{1}^{2} q_{2}^{2} .
\end{aligned}
$$

Here 1 and $\omega=\sqrt{c}$ are the linear normal mode frequencies. When $c=1$ we have a $1: 1$ resonance with

$$
a_{1}=-\frac{d}{6} \quad a_{2}=0 \quad b_{1}=\frac{f}{24} \quad b_{2}=\frac{d}{4} \quad b_{3}=0 .
$$

Substituting into (6.12), we get

$$
\begin{array}{lc}
\alpha_{1}=\frac{1}{192}\left(3 f-5 d^{2}+18 d\right) & \alpha_{2}=-\frac{1}{4} d \\
\beta_{1}=\frac{1}{48}\left(3 f-5 d^{2}-6 d\right) & \beta_{2}=\frac{1}{48}\left(3 f-5 d^{2}\right) .
\end{array}
$$

Thus the non-degeneracy conditions $\alpha_{2} \neq 0, \quad \beta_{1} \neq 0, \alpha_{2}+\beta_{1} \neq 0, \quad \beta_{1} \pm \beta_{2} \neq 0$,

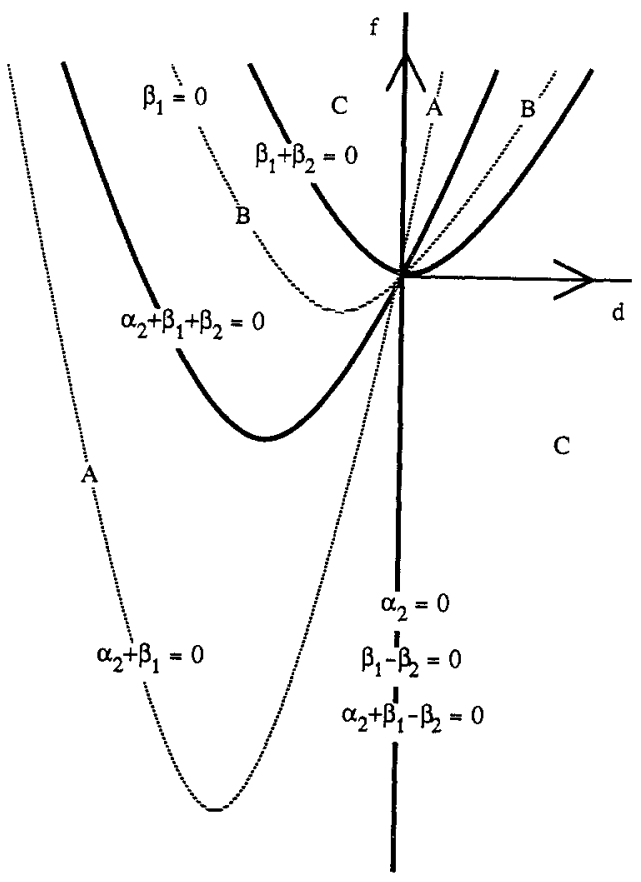

Figure 6. Division of the $(d, f)$ plane by nondegeneracy conditions. The conditions shown by dotted curves do not affect the nonlinear normal modes. The solid curves divide the plane into regions marked $A, B, C$. 
Table 4. Nonlinear normal modes of the spring pendulum. A, B, C refer to regions in figure 6.

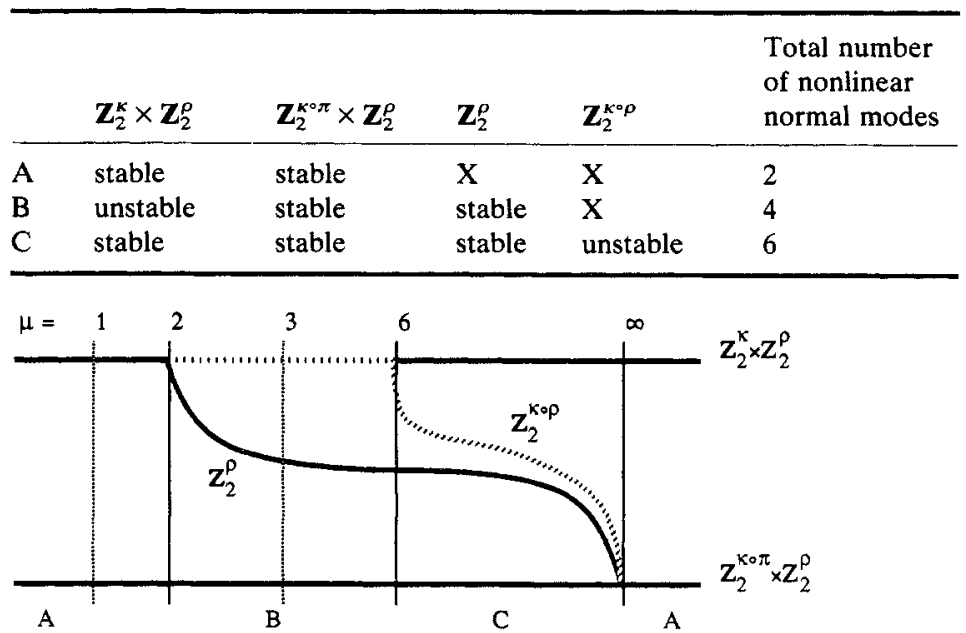

Figure 7. Schematic bifurcation diagram showing group orbits of nonlinear normal modes for the spring pendulum at 1:1 resonance. Each of the $Z_{2}^{\rho}$ and $Z_{2}^{\kappa^{\circ \rho}}$ branches represents two nonlinear normal modes which lie in the same group orbit but whose trajectories have distinct point sets.

$\alpha_{2}+\beta_{1} \pm \beta_{2} \neq 0$ become

$$
d \neq 0 \quad 3 f-5 d^{2} \neq 3 d, 6 d, 9 d, 18 d
$$

or equivalently $\mu \equiv\left(3 f-5 d^{2}\right) / 3 d \neq \infty, 1,2,3,6$. The nonlinear normal modes of the spring pendulum are shown in figure 6 and table 4 . A schematic bifurcation diagram showing group orbits of nonlinear normal modes is given in figure 7 .

Note in particular:

(i) the 'pendulum' mode with symmetry group $\mathbf{Z}_{2}^{\mathrm{K}^{\circ} \pi} \times \boldsymbol{Z}_{2}^{\rho}$ is always stable;

(ii) the $\mathbf{Z}_{2}^{K \circ \rho}$ mode is never stable;

(iii) it is not clear what significance, if any, the non-degeneracy conditions $\alpha+\beta_{1} \neq 0, \beta_{1} \neq 0$ have for the spring pendulum-but theorem 6.3 does not apply to values of $(d, f)$ for which $\alpha_{2}+\beta_{1}=0$ or $\beta_{1}=0$. It may be possible to refine theorem 5.1 to remedy such problems.

If the spring obeys Hooke's Law then all of the non-degeneracy conditions are violated, so our results do not apply. Note that the linear spring does not lead to a linear equation, but is nevertheless highly degenerate. Jan Sanders (private communication) has computed the Birkhoff normal form to higher order in this case, and all higher-order terms that have been computed are zero. It would be interesting to see how far this continues.

\section{Acknowledgments}

The work of James Montaldi was partially supported by an SERC Research Grant and by an NWO Research Grant held at the University of Utrecht. The work of Mark Roberts was supported by an SERC Advanced Research Fellowship. Ian Stewart was supported by an SERC Research Grant. We are grateful to Jim Damon 
for information on unpublished work, and to Yieh-Hei Wan and the referees for suggesting a number of corrections and clarifications.

\section{References}

Abraham R and Marsden J E 1978 Foundations of Mechanics 2nd edn (Reading, MA: Benjamin-Cummings)

Alexander J and Yorke J A 1978 Global bifurcation of periodic orbits Am. J. Math. 100 263-92

Arnold V I 1976 Wave front evolution and equivariant Morse lemma Commun. Pure Appl. Math. 29 557-81

Churchill R C, Krummer M and Rod D L 1983 On averaging, reduction, and symmetry in Hamiltonian systems J. Diff. Eq. 49 359-414

Churchill R C and Lee D 1987 Harmonic oscillators at low energies Preprint Hunter College, CUNY

Damon J 1988 Topological equivalence of bifurcation problems Nonlinearity 1311-32

Duistermaat J J 1984 Bifurcations of periodic solutions near equilibrium points of Hamiltonian systems Bifurcation Theory and Applications, Montecatini 1983 ed L Salvadori (Lecture Notes in Math. 1057) (Berlin: Springer) pp 57-105

Elphick C, Tirapegui E, Brachet M E, Coullet P and Iooss G 1987 A simple global characterisation for normal forms of singular vector fields Physica 29D 95-127

Golubitsky M and Stewart I N 1985 Hopf bifurcation in the presence of symmetry Arch. Rat. Mech. Anal. 87 107-65

1986 Hopf bifurcation with dihedral group symmetry: coupled nonlinear oscillators Multiparameter Bifurcation Theory M Golubitsky and J Guckenheimer (Contemporary Math. 56) (Providence, RI: American Mathematical Society) pp 131-73

Golubitsky M, Stewart I N and Schaeffer D G 1988 Singularities and Groups in Bifurcation Theory vol II (Berlin: Springer)

Kummer M 1986 On resonant Hamiltonian system with finitely many degrees of freedom Local and Global Methods of Nonlinear Dynamics ed A W Saenz, W W Zachary and R Cawley (Lecture Notes in Physics 252) (Berlin: Springer)

Magnus R 1980 A splitting lemma for non-reflexive Banach spaces $M$. Scand. 46 118-28

Montaldi J A, Roberts R M and Stewart I N 1988 Periodic solutions near equilibria of symmetric Hamiltonian systems Phil. Trans. R. Soc. A 325 237-93

1990 Stability of nonlinear normal modes of symmetric Hamiltonian systems Nonlinearity 3

Moser J 1976 Periodic orbits near equilibria and a theorem by Alan Weinstein Commun. Pure Appl. Math. 29 727-47

Roberts R M 1986 Characterisations of finitely determined equivariant map germs Math. Ann. 275 583-97

1988 Nonlinear normal modes of the spring pendulum Preprint University of Warwick

Vanderbauwhede A 1982 Local Bifurcation and Symmetry (Research Notes in Math. 75) (London: Pitman)

1989 Center manifolds, Normal Forms, and Elementary Bifurcations Dynamics Reported vol 2 ed

U Kirchgraber and H O Walther (New York: Wiley) pp 89-169

van der Meer J-C 1985 The Hamiltonian Hopf bifurcation (Lecture Notes in Math. 1160) (Berlin: Springer)

Weinstein A 1973 Normal modes for nonlinear Hamiltonian systems Invent. Math. 20 47-57

1978 Bifurcations and Hamilton's principle Math. Z. 159 235-48 